Pacific

Journal of

Mathematics

ANALYTIC STABILITY OF THE CR CROSS-CAP

\author{
ADAM COFFMAN
}




\title{
ANALYTIC STABILITY OF THE CR CROSS-CAP
}

\author{
ADAM COFFMAN
}

\begin{abstract}
For $m<n$, any real analytic $m$-submanifold of complex $n$-space with a nondegenerate CR singularity is shown to be locally equivalent, under a holomorphic coordinate change, to a fixed real algebraic variety defined by linear and quadratic polynomials. The situation is analogous to Whitney's stability theorem for cross-cap singularities of smooth maps. The complex analyticity of the normalizing transformation is proved using a rapid convergence argument.
\end{abstract}

\section{Introduction}

For $m \leq n$, if a real $m$-manifold $M$ is embedded in $\mathbb{C}^{n}$, then for each point $\boldsymbol{x}$ on $M$ there are two possibilities: the tangent $m$-plane at $\boldsymbol{x}$ may contain a complex line, so $M$ is said to be $C R$ singular at $\boldsymbol{x}$, or it may not, so $M$ is said to be totally real at $\boldsymbol{x}$. This article will consider the local extrinsic geometry of a real analytically embedded $M$ near a CR singular point, in the case when the CR singularity satisfies some natural nondegeneracy properties and $\frac{2}{3}(n+1) \leq m<n$ (so $(m, n)=(4,5)$ is the case of lowest dimension). The main result is an algebraizability property: there exists a holomorphic coordinate change in a neighborhood of $\boldsymbol{x}$ so that $M$ is real algebraic in the new coordinate system. In fact, $M$ will be biholomorphically equivalent to a fixed normal form variety, so that, unlike the well-known $m=n$ case, nondegenerate CR singularities have no continuous invariants under biholomorphisms.

The analysis of normal forms near CR singular points is part of the program of studying the local equivalence problem for real $m$-submanifolds of $\mathbb{C}^{n}$, as described in [Baouendi et al. 2000]. Normal forms for CR singular real $n$-manifolds in $\mathbb{C}^{n}$, where $m=n \geq 2$, have been the subject of much study; see, for example, [Bishop 1965; Moser 1985; Moser and Webster 1983; Webster 1985]. Real surfaces in $\mathbb{C}^{n}$ ( $m=2, n \geq 3$ ) have been considered in [Harris 1981; 1983; Coffman 2004], and real threefolds in $\mathbb{C}^{4}$ in [Coffman 2006]. A formal normal form for a $\mathrm{CR}$ singular real 4-manifold in $\mathbb{C}^{5}$ was found in [Beloshapka 1997] and [Coffman 1997] - it was shown that there exists a transformation (not unique) defined by formal power

MSC2000: 32V40, 32S05.

Keywords: normal form, CR singularity, real submanifold. 
series, taking $M$ to the normal form. The new result here is the existence of a normalizing transformation defined by series that are convergent in a neighborhood of the singularity.

\section{Topological considerations}

We briefly recall some topological properties of CR singularities. We could consider real submanifolds of any complex manifold, but since the main result on the normalization is about the local geometry, we can begin by assuming $M$ is a smoothly immersed real $m$-manifold in $\mathbb{C}^{n}$.

The most basic invariant of a CR singularity at a point $x \in M$ is the number $\boldsymbol{j}(\boldsymbol{x})=\operatorname{dim}_{\mathbb{C}} T_{\boldsymbol{x}} \cap J_{\boldsymbol{x}} T_{\boldsymbol{x}}$, where $T_{\boldsymbol{x}}$ is the real tangent space of $M$ at $\boldsymbol{x}$ and $J_{\boldsymbol{x}}$ is the complex structure operator corresponding to scalar multiplication by $i$ on the tangent space of the ambient complex manifold. The number $\boldsymbol{j}(\boldsymbol{x})$ is the dimension of the largest complex subspace tangent to $M$ at $\boldsymbol{x}$, so $0 \leq \boldsymbol{j}(\boldsymbol{x}) \leq m / 2$.

One way to keep track of $\boldsymbol{j}(\boldsymbol{x})$ is the following construction. For $m \leq n$, let $G$ be the grassmannian variety of real $m$-subspaces in $\mathbb{C}^{n} \cong \mathbb{R}^{2 n}$; see [Garrity 2000; Coffman 1997]. The real $m$-subspaces $T$ such that $\operatorname{dim}_{\mathbb{C}} T \cap i T \geq j$ form a subvariety $\mathscr{D}_{j}$ of real codimension $2 j(n-m+j)$ in $G$. The occurrence of complex tangents of an immersion corresponds to the intersection of $\mathscr{D}_{j}$ with the image of the Gauss map $M \rightarrow G: \boldsymbol{x} \mapsto T_{\boldsymbol{x}}$, and the immersion could be called "generic" if the Gauss map meets each stratum $\mathscr{D}_{j} \backslash \mathscr{D}_{j+1}$ transversely. So, generic immersions of $M$ in $\mathbb{C}^{n}$ are totally real outside a subset of $M$ of codimension $2(n-m+1)$, and if $m<\frac{2}{3}(n+1)$, a generic immersion of $M$ is totally real everywhere. This resembles the bounds in Whitney's embedding and immersion theorems [1944a; 1944b]. In the range $\frac{2}{3}(n+1) \leq m \leq n$, CR singularities are topologically stable - small smooth perturbations of a generic immersion with a CR singular point will still have a CR singular point. For compact real submanifolds of complex manifolds, there are topological obstructions to the property of being totally real at every point, and the CR singularities can be enumerated by characteristic class formulas. See [Domrin 1995a; 1995b; Coffman 1997] and references therein on this topic.

The case addressed by this paper is $\frac{2}{3}(n+1) \leq m<n$, and $\boldsymbol{j}(\boldsymbol{x})=1$; that is, only points $\boldsymbol{x}$ where exactly one complex line is tangent at $\boldsymbol{x}$ will be considered, and only in dimension cases where the CR singularity is stable under smooth perturbations of the immersion. As mentioned in the Introduction, the $m=n$ case has a qualitatively different local geometry than the $m<n$ case and is not considered here. The cases $(m, n)=(2,3)$ or $(3,4)$, considered in [Coffman 2004; 2006], fall outside the topological stability range. The case $(m, n)=(4,5)$, considered in [Beloshapka 1997; Domrin 1995a; Coffman 1997; 2002], has the lowest dimensions in the range, and the generic singularity is isolated (codimension 4 in $M$ ). 


\section{The quadratic normal form}

Let the ambient complex space be $\mathbb{C}^{n}$, with coordinates $\left(z_{1}, \ldots, z_{n}\right)$. The real and imaginary parts of the coordinate functions are labeled $z_{j}=x_{j}+i y_{j}$ for $j=$ $1, \ldots, n$. Let $M$ be a real analytic $m$-dimensional submanifold embedded in $\mathbb{C}^{n}$, with $m<n$, and let $\boldsymbol{x}$ be a point on $M$ at which $M$ is tangent to a complex line but not to any complex 2-plane - in terms of the previous section, $\boldsymbol{j}(\boldsymbol{x})=1$, which we regard as a nondegeneracy assumption, since for $M$ in general position, the points where $\boldsymbol{j}(\boldsymbol{x})>1$ form a subset of higher codimension.

By a translation that moves $x$ to the origin $\overrightarrow{0}$, and then a complex linear transformation of $\mathbb{C}^{n}$, the tangent space $T=T_{\overrightarrow{0}}$ of $M$ can be assumed to be the one spanned by $\left(x_{1}, y_{1}, x_{2}, \ldots, x_{m-1}\right)$, and thus to contain the $z_{1}$-axis. Then there is some neighborhood $\Delta$ of the origin in $\mathbb{C}^{n}$ so that the defining equations of $M$ in $\Delta$ are in the form of a graph over a neighborhood of the origin in $T$ :

$$
\begin{aligned}
y_{s} & =H_{s}\left(z_{1}, \bar{z}_{1}, x_{2}, \ldots, x_{m-1}\right) \\
z_{u} & =h_{u}\left(z_{1}, \bar{z}_{1}, x_{2}, \ldots, x_{m-1}\right),
\end{aligned}
$$

where $H_{s}$, for $s=2, \ldots, m-1$, is a real-valued real analytic function, and $h_{u}$, for $u=m, \ldots, n$, is a complex-valued real analytic function, with $H_{s}$ and $h_{u}$ defined in a neighborhood of the origin in $T$, and vanishing to second order at $\left(x_{1}, y_{1}, x_{2}, \ldots, x_{m-1}\right)=(0, \ldots, 0)$. The expression " $x_{2}, \ldots, x_{m-1}$ " is abbreviated as just $x$. So, the defining functions are of the following form:

$$
\begin{array}{r}
H_{s}\left(z_{1}, \bar{z}_{1}, x\right)=\alpha_{s} z_{1}^{2}+\beta_{s} z_{1} \bar{z}_{1}+\gamma_{s} \bar{z}_{1}^{2}+\sum \delta_{s}^{s_{1}} z_{1} x_{s_{1}}+\sum \epsilon_{s}^{s_{1}} \bar{z}_{1} x_{s_{1}}+ \\
+\theta_{s} \theta_{s}^{s_{1} s_{2}} x_{s_{1}} x_{s_{2}} \\
\left.+z_{1}, \bar{z}_{1}, x\right), \\
h_{u}\left(z_{1}, \bar{z}_{1}, x\right)=\alpha_{u} z_{1}^{2}+\beta_{u} z_{1} \bar{z}_{1}+\gamma_{u} \bar{z}_{1}^{2}+\sum \delta_{u}^{s_{1}} z_{1} x_{s_{1}}+\sum \epsilon_{u}^{s_{1}} \bar{z}_{1} x_{s_{1}}+ \\
+\sum \theta_{u}^{s_{1} s_{2}} x_{s_{1}} x_{s_{2}} \\
+e_{u}\left(z_{1}, \bar{z}_{1}, x\right),
\end{array}
$$

with $E_{s}, e_{u}$ having terms of degree three or higher. Each of these functions can be expressed as the restriction to $\left\{\left(z_{1}, \zeta, x\right) \in \mathbb{C}^{m}: \zeta=\bar{z}_{1}, x=\bar{x}\right\}$ of an $m$-variable series with complex coefficients:

$$
\begin{aligned}
& H_{s}\left(z_{1}, \zeta, x\right)=\alpha_{s} z_{1}^{2}+\beta_{s} z_{1} \zeta+\gamma_{s} \zeta^{2}+\sum \delta_{s}^{s_{1}} z_{1} x_{s_{1}}+\sum \epsilon_{s}^{s_{1}} \zeta x_{s_{1}} \\
& +\sum \theta_{s}^{s_{1} s_{2}} x_{s_{1}} x_{s_{2}}+\sum_{a+b+I \geq 3} E_{s}^{a b I} z_{1}^{a} \zeta^{b} x^{I} \\
& h_{u}\left(z_{1}, \zeta, x\right)=\alpha_{u} z_{1}^{2}+\beta_{u} z_{1} \zeta+\gamma_{u} \zeta^{2}+\sum \delta_{u}^{s_{1}} z_{1} x_{s_{1}}+\sum \epsilon_{u}^{s_{1}} \zeta x_{s_{1}} \\
& +\sum \theta_{u}^{s_{1} s_{2}} x_{s_{1}} x_{s_{2}}+\sum_{a+b+I \geq 3} e_{u}^{a b I} z_{1}^{a} \zeta^{b} x^{I},
\end{aligned}
$$


where $x^{I}$ abbreviates $x_{2}^{i_{2}} x_{3}^{i_{3}} \cdots x_{m-1}^{i_{m-1}}$ and $a+b+I$ abbreviates $a+b+i_{2}+\ldots+i_{m-1}$. Each of the series in $\left(z_{1}, \zeta, x\right)$ converges on some set of the form

$$
\left\{\left(z_{1}, \zeta, x\right):\left|z_{1}\right|<r,|\zeta|<r,\left|x_{s}\right|<r\right\}
$$

with $r>0$, to a complex analytic function, with $\gamma_{s}=\bar{\alpha}_{s}, \epsilon_{s}^{s_{1}}=\overline{\delta_{2}^{s_{1}}}$, etc., so that $H_{s}\left(z_{1}, \bar{z}_{1}, x\right)$ and $E_{s}\left(z_{1}, \bar{z}_{1}, x\right)$ are real-valued.

Definition 3.1. A (formal) monomial $C z_{1}^{a} \zeta^{b} x^{I}$ (with complex coefficient $C$ ) has degree $a+b+I$. A (convergent or formal) power series in $m$ variables, say $e\left(z_{1}, \zeta, x\right)=\sum e^{a b I} z_{1}^{a} \zeta^{b} x^{I}$, is said to have degree $d$ if $e^{a b I}=0$ for all $(a, b, I)$ such that $a+b+I<d$. Sometimes a series of degree $d$ will be abbreviated $O(d)$. An ordered $k$-tuple of series $\left(e_{1}, \ldots, e_{k}\right)$ has degree $d$ if all its components have degree $d$.

Definition 3.2. Similarly for $n$ variables, a monomial $C z_{1}^{a_{1}} \cdots z_{n}^{a_{n}}$ has degree $a_{1}+$ $\cdots+a_{n}$, but we will also work with the weight $a_{1}+\cdots+a_{m-1}+2 a_{m}+\cdots+2 a_{n}$. A series $p(\vec{z})=\sum p^{a_{1} \ldots a_{n}} z_{1}^{a_{1}} \ldots z_{n}^{a_{n}}$ has weight $W$ if $p^{a_{1} \ldots a_{n}}=0$ when $a_{1}+\cdots+$ $a_{m-1}+2 a_{m}+\cdots+2 a_{n}<W$.

We consider two coordinate systems for a neighborhood of the origin in $\mathbb{C}^{n}$ : the previously mentioned $\vec{z}=\left(z_{1}, \ldots, z_{n}\right)$, and a new system $\tilde{z}=\left(\tilde{z}_{1}, \ldots, \tilde{z}_{n}\right)$, with $\tilde{z}_{j}=\tilde{x}_{j}+\tilde{y}_{j}$. The two systems are related by the change of coordinates

$$
\tilde{z}=\vec{z}+\vec{p}(\vec{z}),
$$

where $\vec{p}(\vec{z})=\left(p_{1}(\vec{z}), \ldots, p_{n}(\vec{z})\right)$ and each component $p_{j}$ is a holomorphic function of $z_{1}, \ldots, z_{n}$ whose series expansion has weight 2 , and for $j \geq m$, also degree 2 . Such a transformation of $\mathbb{C}^{n}$ has invertible linear part, so it is invertible on some neighborhood of $\overrightarrow{0}$. In the calculations of this section, we will neglect considering the size of that neighborhood, and consider only points close enough to the origin, but the size of the domain of $\vec{p}$ will be important information in later sections.

The goal of this section is to establish some nondegeneracy conditions on the defining equations (1), by using complex linear transformations and nonlinear transformations of the form (2) to put the quadratic terms of (1) into a normal form. Similar calculations have already been done in the case $m=n$ and the cases $(m, n)=(2,3),(3,4)$, and $(4,5)$, in [Bishop 1965; Coffman 2004; 2006; Beloshapka 1997], respectively, so we will skip some of the computational details.

As the first special case of a transformation of the form (2) to be used, let $p_{1}=0$ and let $p_{2}, \ldots, p_{n}$ be homogeneous quadratic polynomials in $z_{1}, \ldots, z_{m-1}$. Using such a transformation, the quadratic terms in $h_{u}$ that are products of $z_{1}$ and $x$ only, without a $\bar{z}_{1}$ factor, can be eliminated in the new coordinate system, or their coefficients $\left(\alpha_{u}, \delta_{u}^{s_{1}}, \theta_{u}^{s_{1} s_{2}}\right)$ can be altered to attain any complex values, by a suitable choice of $\vec{p}$. This transformation may also change some higher-degree 
terms but does not alter the coefficients $\beta_{u}, \gamma_{u}, \epsilon_{u}^{s_{1}}$. Similarly, the quadratic terms without $\bar{z}_{1}$ in each $H_{s}$ can also be eliminated by a transformation $\tilde{z}=\vec{z}+\vec{p}$, which simultaneously eliminates their conjugates (using $\gamma_{s}=\bar{\alpha}_{s}$ ), leaving only the mixed term $\beta_{s} z_{1} \bar{z}_{1}$.

The result of this preliminary normalization is that for any CR singular submanifold $M$ of the form (1), there exists a quadratic coordinate transformation of the form (2) with $p_{1}=0$, so that $M$ has the following general normal form. In a local coordinate system $\vec{z}$ in some neighborhood of the CR singularity, the defining equations of $M$ are of the form (1), with

$$
\begin{aligned}
& y_{s}=H_{s}\left(z_{1}, \bar{z}_{1}, x\right)=\beta_{s} z_{1} \bar{z}_{1}+O(3), \\
& z_{u}=h_{u}\left(z_{1}, \bar{z}_{1}, x\right)=\beta_{u} z_{1} \bar{z}_{1}+\gamma_{u} \bar{z}_{1}^{2}+\sum \epsilon_{u}^{s_{1}} \bar{z}_{1} x_{s_{1}}+O(3) .
\end{aligned}
$$

At this point we consider which invertible complex linear transformations of $\mathbb{C}^{n}$ fix the tangent plane $T$ with coordinates $\left(z_{1}, x\right)$. The matrix representation of such a transformation must be of the form $\tilde{z}=\boldsymbol{A} z$, where

$$
\boldsymbol{A}=\left(\begin{array}{ccc}
a_{1} & a_{2} \ldots a_{m-1} & a_{m} \ldots a_{n} \\
0 & \boldsymbol{R} & * \\
0 & 0 & \boldsymbol{C}
\end{array}\right)
$$

The entries $a_{1}, \ldots, a_{n}$ are complex, with $a_{1} \neq 0$, the $(m-2) \times(m-2)$ block $\boldsymbol{R}$ has real entries and a nonzero determinant, and the $(n-m+1) \times(n-m+1)$ block $\boldsymbol{C}$ has complex entries and a nonzero determinant.

The first nondegeneracy condition is that the $(n-m+1) \times 2$ block of coefficients $\beta_{u}, \gamma_{u}$ in the functions $h_{u}$ satisfies

$$
\operatorname{rank}\left(\begin{array}{cc}
\beta_{m} & \gamma_{m} \\
\vdots & \vdots \\
\beta_{n} & \gamma_{n}
\end{array}\right)=2
$$

In particular, this requires $m<n$. In this nondegenerate case, there is a linear transformation of $\mathbb{C}^{n}$ which uses the block $\boldsymbol{C}$ in the complex matrix above to put these coefficients into a row echelon form:

$$
\begin{aligned}
z_{t}=h_{t}\left(z_{1}, \bar{z}_{1}, x\right) & =\sum \epsilon_{t}^{s_{1}} \bar{z}_{1} x_{s_{1}}+O(3), \\
z_{n-1}= & h_{n-1}\left(z_{1}, \bar{z}_{1}, x\right)=\bar{z}_{1}^{2}+\sum \epsilon_{n-1}^{s_{1}} \bar{z}_{1} x_{s_{1}}+O(3), \\
z_{n}=h_{n}\left(z_{1}, \bar{z}_{1}, x\right) & =z_{1} \bar{z}_{1}+\sum \epsilon_{n}^{s_{1}} \bar{z}_{1} x_{s_{1}}+O(3),
\end{aligned}
$$

for $t=m \ldots, n-2$, or there are no $h_{t}$ expressions if $m=n-1$.

As a consequence of the first nondegeneracy condition on the functions $h_{u}$, the functions $H_{s}$ can also be simplified. There is a linear transformation with 
components $\tilde{z}_{s}=z_{s}-i \beta_{s} z_{n}$ (where for $s=2, \ldots, m-1$, the complex coefficients $-i \beta_{s}$ are entries from the $*$ block of the matrix $\boldsymbol{A}$ ) that eliminates the $\beta_{s} z_{1} \bar{z}_{1}$ terms from each function $H_{s}$. This may introduce more terms of the form $z_{1} x_{s_{1}}$ or $\bar{z}_{1} x_{s_{1}}$ in the $H_{s}$ functions, which can be eliminated by $\tilde{z}_{s}=z_{s}+p_{s}$ quadratic transformations as done previously, without reintroducing any $z_{1} \bar{z}_{1}$ terms.

A linear transformation of the form $\tilde{z}_{1}=z_{1}+\sum a_{s} z_{s}$, using the block $a_{2}, \ldots$, $a_{m-1}$ from the matrix $\boldsymbol{A}$, can eliminate the terms of the form $\bar{z}_{1} x_{s_{1}}$ from either the $h_{n-1}$ quantity or the $h_{n}$ quantity, but generally not both at once - we make the choice to eliminate the terms $\sum \epsilon_{n-1}^{s_{1}} \bar{z}_{1} x_{s_{1}}$ from $h_{n-1}$. This may introduce more terms of the form $z_{1} x_{s_{1}}$ or $x_{s_{1}} x_{s_{2}}$ in the other $h_{u}$ functions, which can be eliminated by $\tilde{z}_{u}=z_{u}+p_{u}$ quadratic transformations as done previously.

The real and imaginary parts of the coefficients $\epsilon_{u}^{s_{1}}$, for $u=m, \ldots, n-2$ and $u=n$, on the terms $\bar{z}_{1} x_{s_{1}}, s_{1}=2, \ldots, m-1$, form a real $2(n-m) \times(m-2)$ matrix, in this expression where the left-hand side is a column $(n-m)$-vector:

$$
\begin{aligned}
& \left(\sum \epsilon_{u}^{s_{1}} \bar{z}_{1} x_{s_{1}}\right)_{u=m, \ldots, n-2, n} \\
& \quad=\left(\begin{array}{ccccc}
1 & i & \ldots & 0 & 0 \\
\vdots & & & & \vdots \\
0 & 0 & \ldots & 1 & i
\end{array}\right)\left(\begin{array}{ccccc}
\operatorname{Re} \epsilon_{m}^{2} & \operatorname{Re} \epsilon_{m}^{3} & \ldots & \operatorname{Re} \epsilon_{m}^{m-1} \\
\operatorname{Im} \epsilon_{m}^{2} & \operatorname{Im} \epsilon_{m}^{3} & \ldots & \operatorname{Im} \epsilon_{m}^{m-1} \\
\vdots & & & \vdots \\
\operatorname{Re} \epsilon_{n-2}^{2} & \operatorname{Re} \epsilon_{n-2}^{3} & \ldots & \operatorname{Re} \epsilon_{n-2}^{m-1} \\
\operatorname{Im} \epsilon_{n-2}^{2} & \operatorname{Im} \epsilon_{n-2}^{3} & \ldots & \operatorname{Im} \epsilon_{n-2}^{m-1} \\
\operatorname{Re} \epsilon_{n}^{2} & \operatorname{Re} \epsilon_{n}^{3} & \ldots & \operatorname{Re} \epsilon_{n}^{m-1} \\
\operatorname{Im} \epsilon_{n}^{2} & \operatorname{Im} \epsilon_{n}^{3} & \ldots & \operatorname{Im} \epsilon_{n}^{m-1}
\end{array}\right)\left(\begin{array}{c}
x_{2} \\
\vdots \\
x_{m-1}
\end{array}\right) \bar{z}_{1} .
\end{aligned}
$$

The second nondegeneracy condition is that this real matrix has rank $2(n-m)$. It follows that the number of $x_{s}$ directions, $m-2$, must be greater than or equal to the number $2(n-m)$, and this is equivalent to $m \geq \frac{2}{3}(n+1)$, exactly the lower bound of the dimensions of topological stability, as discussed in Section 2.

When the second nondegeneracy condition holds, the real $\boldsymbol{R}$ block of the matrix $\boldsymbol{A}$ can transform the $x_{s}$ variables to put the real matrix above into echelon form, transforming the real and imaginary parts of the $\epsilon_{u}^{s_{1}}$ coefficients, without altering the $\bar{z}_{1}^{2}$ and $z_{1} \bar{z}_{1}$ terms. We get the following quadratic normal form for a nondegenerate CR singularity:

$$
\begin{aligned}
y_{s} & =H_{s}\left(z_{1}, \bar{z}_{1}, x\right)=E_{s}\left(z_{1}, \bar{z}_{1}, x\right)=O(3), \\
z_{t} & =h_{t}\left(z_{1}, \bar{z}_{1}, x\right)=\bar{z}_{1} x_{2(t-m+2)}+i \bar{z}_{1} x_{2(t-m+2)+1}+e_{t}\left(z_{1}, \bar{z}_{1}, x\right), \\
z_{n-1}= & h_{n-1}\left(z_{1}, \bar{z}_{1}, x\right)=\bar{z}_{1}^{2}+e_{n-1}\left(z_{1}, \bar{z}_{1}, x\right), \\
z_{n}=h_{n}\left(z_{1}, \bar{z}_{1}, x\right) & =z_{1} \bar{z}_{1}+\bar{z}_{1} x_{2}+i \bar{z}_{1} x_{3}+e_{n}\left(z_{1}, \bar{z}_{1}, x\right),
\end{aligned}
$$


with $s=2, \ldots, m-1, t=m, \ldots, n-2$, or, again, there are no $h_{t}$ expressions if $m=n-1$. If $m>\frac{2}{3}(n+1)$, then the $x_{2}, \ldots, x_{2 n-2 m+1}$ variables appear in the quadratic part of the normal form but the variables $x_{2 n-2 m+2}, \ldots, x_{m-1}$ do not. In fact, near the origin, the locus of CR singularities (with $\boldsymbol{j}(\boldsymbol{x})=1$ ) is a codimension $2(n-m+1)$ submanifold of $M$ whose tangent space at the origin is the real subspace with coordinates $x_{2 n-2 m+2}, \ldots, x_{m-1}$.

Having stated these two nondegeneracy conditions, we are now ready to state the main result:

Proposition 3.3. Given $\frac{2}{3}(n+1) \leq m<n$, let $M$ be a real analytic $m$-submanifold of $\mathbb{C}^{n}$ with a $C R$ singularity at $\boldsymbol{x}$, with $\boldsymbol{j}(\boldsymbol{x})=1$. If its local defining equations (of the form (1)) satisfy both nondegeneracy conditions (the full rank of the coefficient matrices (3), (4)) so that they can be put into the form (5), then there exists a holomorphic coordinate change $\tilde{z}=\vec{z}+\vec{p}$ as in (2), in a neighborhood of $\overrightarrow{0} \in \mathbb{C}^{n}$, transforming the equations (5) into the real algebraic normal form

$$
\begin{array}{rlrl}
\tilde{y}_{s} & =0 & \text { for } s=2 \ldots, m-1, \\
\tilde{z}_{t} & =\overline{\tilde{z}}_{1}\left(\tilde{x}_{2(t-m+2)}+i \tilde{x}_{2(t-m+2)+1}\right) & \text { for } t=m \ldots, n-2, \\
\tilde{z}_{n-1} & =\overline{\tilde{z}}_{1}^{2}, & \\
\tilde{z}_{n} & =\overline{\tilde{z}}_{1}\left(\tilde{z}_{1}+\tilde{x}_{2}+i \tilde{x}_{3}\right) . & &
\end{array}
$$

The real algebraic variety defined by (6) is denoted $\widetilde{M}^{m, n}$, or more briefly $\tilde{M}$. The example $\widetilde{M}^{4,5}$ is exactly the normal form of [Beloshapka 1997]. The proposition states that any real analytic $M$ satisfying only $\boldsymbol{j}(\boldsymbol{x})=1$ at a point and both quadratic nondegeneracy conditions is locally biholomorphically equivalent to the real algebraic model. This is the "analytic stability" mentioned in the title, and it is apparently analogous to stability theorems in the singularity theory of smooth maps, where any sufficiently nondegenerate singularity is equivalent under a change of coordinates to a unique polynomial model. The equations for $\tilde{M}$ resemble the normal forms for smooth maps with cross-cap (or " $S_{1}$ ") singularities, as in [Whitney 1958; Haefliger 1961; Golubitsky and Guillemin 1973, §VII.4], and $\widetilde{M}$ and the images of the singular maps also have similar structures as a cartesian product when the singularity is not isolated. The main difference between Whitney's normal forms and (6) is that the quantities in (6) are not monomials, and cannot be simultaneously transformed into monomials by holomorphic coordinate transformations in the nondegenerate case. More will be said about the analogies with singularity theory in Section 8 .

In the case $m=\frac{2}{3}(n+1)$ when the singularity is isolated, some of the topological invariants mentioned in Section 2 depend on an orientation of $M$, so it may be useful to consider normalizing transformations that fix a given orientation of the 
tangent plane $T$. This corresponds to the real block $\boldsymbol{R}$ of matrix $\boldsymbol{A}$ having a positive determinant, and the last equation of the normal form (5) falls into two cases: $z_{n}=$ $\bar{z}_{1}\left(z_{1}+x_{2} \pm i x_{3}\right)$. The two normal forms are equivalent under the biholomorphic transformation $\tilde{z}_{3}=-z_{3}$, but this reverses the orientation of $T$. In the remaining sections we will not be concerned with the orientation.

\section{A functional equation}

To show the existence of a normalizing transformation, we will set up a system of nonlinear functional equations, so that any solution $\vec{p}$ of the system will define a normalizing transformation $\tilde{z}=\vec{z}+\vec{p}$ as in (2). In addition to finding a formal power series solution, we will also have to show that the solution is convergent in some neighborhood of the origin. The method of proof is the rapid convergence technique, as used in [Moser 1985] and [Coffman 2004]. Rather than trying to solve the system of equations directly, we first find an approximate solution by solving a related system of linear equations. Iteration of this process gives a sequence of approximations that approach an exact solution. The issue of the domain of convergence of the exact solution was not addressed by [Beloshapka 1997], and was left open in [Coffman 1997]. In this latter paper, each approximate solution in the sequence was constructed only on a domain a fraction of the size of the previous one in the sequence - when the domains shrink to a point, the limit is an exact formal series solution, but no conclusion can be drawn about its analyticity. The new step here, which is crucial for the method of [Moser 1985] to be applicable, is the construction of a sequence of approximate solutions whose domains shrink slowly enough so their diameters are bounded below by a positive constant.

Starting with the quadratic part of the defining equations in normal form (5), we consider the effect of a coordinate change (2). As previously mentioned, the $\tilde{z}=\vec{z}+\vec{p}$ transformation is (at least formally) invertible near $\overrightarrow{0}$, and it may be useful to think of $\tilde{z}=\vec{z}+\vec{p}$ as having identity linear part, although there could be linear terms with weight 2 , for example, $\tilde{z}_{1}=z_{1}+a_{n} z_{n}$.

In terms of $\tilde{z}$ and $\vec{z}$, consider the system of equations

$$
\begin{aligned}
& 0=\operatorname{Im}\left(\tilde{z}_{s}\right)=\operatorname{Im}\left(z_{s}+p_{s}(\vec{z})\right), \\
& 0=\tilde{z}_{t}-\left(\overline{\tilde{z}}_{1} \tilde{x}_{2(t-m+2)}+i \overline{\tilde{z}}_{1} \tilde{x}_{2(t-m+2)+1}\right), \\
& =z_{t}+p_{t}(\vec{z})-\overline{\left(z_{1}+p_{1}(\vec{z})\right)} \operatorname{Re}\left(z_{2(t-m+2)}+p_{2(t-m+2)}(\vec{z})\right) \\
& \quad \quad-i \overline{\left(z_{1}+p_{1}(\vec{z})\right)} \operatorname{Re}\left(z_{2(t-m+2)+1}+p_{2(t-m+2)+1}(\vec{z})\right), \\
& 0=\tilde{z}_{n-1}-\overline{\tilde{z}}_{1}^{2}=z_{n-1}+p_{n-1}(\vec{z})-\overline{\left(z_{1}+p_{1}(\vec{z})\right)}, \\
& 0=\tilde{z}_{n}-\overline{\tilde{z}}_{1}\left(\tilde{z}_{1}+\tilde{x}_{2}+i \tilde{x}_{3}\right) \\
& =z_{n}+p_{n}(\vec{z})-\overline{\left(z_{1}+p_{1}(\vec{z})\right)}\left(z_{1}+p_{1}(\vec{z})+\operatorname{Re}\left(z_{2}+p_{2}(\vec{z})\right)+i \operatorname{Re}\left(z_{3}+p_{3}(\vec{z})\right)\right) .
\end{aligned}
$$


In order to get (6) to be the defining equations for $M$ in the $\tilde{z}$ coordinates, the preceding equalities must hold for points $\vec{z}$ on $M$ and near $\overrightarrow{0}$. So, we can replace the $\vec{z}=\left(z_{1}, \ldots, z_{n}\right)$ expressions in (7) by the defining functions (5):

$$
\vec{z}=\left(z_{1}, x_{2}+i H_{2}\left(z_{1}, \bar{z}_{1}, x\right), \ldots, h_{n}\left(z_{1}, \bar{z}_{1}, x\right)\right),
$$

to get a system of equations where the right-hand side functions depend only on $z_{1}, \bar{z}_{1}, x$ :

$$
\begin{aligned}
& \begin{array}{l}
0=\operatorname{Im}\left(x_{s}+i H_{s}+p_{s}(\vec{z})\right)=E_{s}\left(z_{1}, \bar{z}_{1}, x\right)+\operatorname{Im} p_{s}(\vec{z}) \\
\begin{array}{r}
0=e_{t}\left(z_{1}, \bar{z}_{1}, x\right)+p_{t}(\vec{z})-\overline{p_{1}(\vec{z})}\left(x_{2(t-m+2)}+i x_{2(t-m+2)+1}\right) \\
-\bar{z}_{1}\left(\operatorname{Re} p_{2(t-m+2)}(\vec{z})+i \operatorname{Re} p_{2(t-m+2)+1}(\vec{z})\right)
\end{array} \\
\quad-\overline{p_{1}(\vec{z})}\left(\operatorname{Re} p_{2(t-m+2)}(\vec{z})+i \operatorname{Re} p_{2(t-m+2)+1}(\vec{z})\right), \\
\begin{array}{r}
0=e_{n-1}\left(z_{1}, \bar{z}_{1}, x\right)+p_{n-1}(\vec{z})-2 \bar{z}_{1} \overline{p_{1}(\vec{z})}-{\overline{p_{1}(\vec{z})}}^{2} \\
0=e_{n}\left(z_{1}, \bar{z}_{1}, x\right)+p_{n}(\vec{z})-\bar{z}_{1}\left(p_{1}(\vec{z})+\operatorname{Re} p_{2}(\vec{z})+i \operatorname{Re} p_{3}(\vec{z})\right)
\end{array} \\
\quad-\overline{p_{1}(\vec{z})}\left(z_{1}+x_{2}+i x_{3}\right)-\overline{p_{1}(\vec{z})}\left(p_{1}(\vec{z})+\operatorname{Re} p_{2}(\vec{z})+i \operatorname{Re} p_{3}(\vec{z})\right) .
\end{array}
\end{aligned}
$$

The components of $\vec{e}=\left(E_{2}, \ldots, E_{m-1}, e_{m}, \ldots, e_{n}\right)$ appear in two ways - as terms in each equation of (9), and also in the $\vec{z}$ input (8) for each $p_{j}(\vec{z})$ in (9), $j=$ $1, \ldots, n$. So, given $\vec{e}$, if we happen to have an exact solution $\vec{p}$ of the system of functional equations above, the conclusion of Proposition 3.3 holds and we are done. However, (9) is a nonlinear system in the unknown quantity $\vec{p}$, where in addition to the composition with the given defining functions (8), there are products of the components $p_{j}$ and their complex conjugates.

As a first step in solving for $\vec{p}$ in terms of $\vec{e}$, consider the system of simpler equations:

$$
\begin{aligned}
& 0=E_{s}\left(z_{1}, \bar{z}_{1}, x\right)+\operatorname{Im} p_{s}(\vec{z}), \\
& 0=e_{t}\left(z_{1}, \bar{z}_{1}, x\right)+p_{t}(\vec{z})-\overline{p_{1}(\vec{z})}\left(x_{2(t-m+2)}+i x_{2(t-m+2)+1}\right) \\
& -\bar{z}_{1}\left(\operatorname{Re} p_{2(t-m+2)}(\vec{z})+i \operatorname{Re} p_{2(t-m+2)+1}(\vec{z})\right), \\
& 0=e_{n-1}\left(z_{1}, \bar{z}_{1}, x\right)+p_{n-1}(\vec{z})-2 \bar{z}_{1} \overline{p_{1}(\vec{z})}, \\
& 0=e_{n}\left(z_{1}, \bar{z}_{1}, x\right)+p_{n}(\vec{z}) \\
& -\bar{z}_{1}\left(p_{1}(\vec{z})+\operatorname{Re} p_{2}(\vec{z})+i \operatorname{Re} p_{3}(\vec{z})\right)-\overline{p_{1}(\vec{z})}\left(z_{1}+x_{2}+i x_{3}\right),
\end{aligned}
$$

where the $\vec{z}$ input for each $p_{j}$ is

$$
\vec{z}=\left(z_{1}, x_{2}, \ldots, x_{m-1}, \bar{z}_{1}\left(x_{4}+i x_{5}\right), \ldots, \bar{z}_{1}^{2}, \bar{z}_{1}\left(z_{1}+x_{2}+i x_{3}\right)\right) .
$$

This simplifies $p_{j}(\vec{z})$ by considering only the linear and quadratic parts of the input (8). Also, the products of $p_{j}$ are dropped, so that these are (real) linear equations. 
To see how the new equations are related to the original system, suppose $\vec{e}$ has degree $d \geq 3$, and that $\vec{p}$ is a solution of (10)-(11) so that $p_{1}, \ldots, p_{2 n-2 m+1}$ have weight $\geq d-1$, and $p_{2 n-2 m+2}, \ldots, p_{n}$ have weight $\geq d$. Evaluating the righthand side of (9) with this solution for $\vec{p}$ evidently results in expressions of degree $\geq 2 d-2$. Converting these expressions in $z_{1}, \bar{z}_{1}, x$ to $\tilde{z}_{1}, \overline{\tilde{z}}_{1}, \tilde{x}$ and equating them to the $\tilde{z}$ expressions in (7) gives the higher-order terms of the new defining equations for $M$ in the $\tilde{z}$ coordinate system. (It will be shown later (Theorem 6.5 ) that in fact for $\vec{z} \in M$ close enough to $\overrightarrow{0}, z_{1}, \bar{z}_{1}, x$ are real analytic functions of $\tilde{z}_{1}, \overline{\tilde{z}}_{1}, \tilde{x}$.) So, while a solution $\vec{p}$ of the linearized equations is just an approximation to the solution of the original system, using such a $\vec{p}$ to define a coordinate transformation does have the effect of nearly doubling the order of vanishing of the $\vec{e}$ quantity.

\section{A solution of the linear equation}

The goal of this section is to construct a solution $\vec{p}$ of the system of linear equations (10)-(11), given the higher-order terms of the defining equations, $\vec{e}$. Considering $\vec{p}$ and $\vec{e}$ as formal power series, such a solution exists but is not unique - this fact, together with the approximate doubling of the degree mentioned in the previous section and iteration of the linearization procedure, is enough to show the (already known, as mentioned previously) formal equivalence of $M$ and $\widetilde{M}$. The solution $\vec{p}$ constructed here will be an $n$-tuple of series in $\vec{z}=\left(z_{1}, \ldots, z_{n}\right)$ with the following properties: the size of the domain of convergence of $\vec{p}$ is comparable in a certain sense to the size of the domain of $\vec{e}$, and also a suitable norm of $\vec{p}$ is bounded in terms of a suitable norm of $\vec{e}$.

Notation 5.1. For $\boldsymbol{r}=\left(r_{1}, \ldots, r_{N}\right) \in \mathbb{R}^{N}$, with all $r_{j}>0$, define a polydisc in $\mathbb{C}^{N}$ by

$$
\mathbb{D}_{\boldsymbol{r}}=\left\{\left(z_{1}, \ldots, z_{N}\right):\left|z_{j}\right|<r_{j}\right\}
$$

As special cases, let

$$
D_{r}=\mathbb{D}_{(r, r, \ldots, r)} \subseteq \mathbb{C}^{m} \quad \text { and } \quad \Delta_{r}=\mathbb{D}_{\left(r, \ldots, r, 2 r^{2}, \ldots, 2 r^{2}, r^{2}, 3 r^{2}\right)} \subseteq \mathbb{C}^{n},
$$

where there are $m-1$ radius lengths $r$ and $n-m-1$ radius lengths $2 r^{2}$, in the $z_{m}, \ldots, z_{n-2}$ coordinate directions.

The initial assumption on the defining equations is that

$$
\vec{e}\left(z_{1}, \bar{z}_{1}, x\right)=\left(E_{2}, \ldots, E_{m-1}, e_{m}, \ldots, e_{n}\right)
$$

is real analytic, so there is some $r>0$ so that each component of $\vec{e}$ is the restriction to $\left\{\zeta=\bar{z}_{1}, x=\bar{x}\right\}$ of a multivariable power series in $\left(z_{1}, \zeta, x\right)$ with center $(0,0, \ldots, 0)$ and complex coefficients which converges on a complex polydisc $D_{r} \subseteq \mathbb{C}^{m}$ (or, equivalently, a complex analytic function on $D_{r}$ ). 
Notation 5.2. For a complex-valued function $e\left(z_{1}, \zeta, x\right)$ of $m$ complex variables, which is defined on some set containing the polydisc $D_{r}$, define the norm

$$
|e|_{r}=\sup _{\left(z_{1}, \zeta, x\right) \in D_{r}}\left|e\left(z_{1}, \zeta, x\right)\right| .
$$

For an $(n-1)$-tuple $\vec{e}=\left(E_{2}, \ldots, e_{n}\right)$, define

$$
|\vec{e}|_{r}=\left|E_{2}\right|_{r}+\cdots+\left|e_{n}\right|_{r} .
$$

For a complex-valued function $p\left(z_{1}, \ldots, z_{n}\right)$ of $n$ complex variables, which is defined on some set containing the polydisc $\Delta_{r}$, define the norm

$$
\|p\|_{r}=\sup _{\vec{z} \in \Delta_{r}}|p(\vec{z})| .
$$

With this notation, we can further assume $r>0$ is small enough so that $\left|\vec{e}\left(z_{1}, \zeta, x\right)\right|_{r}$ is finite. Given $\vec{e}$ with degree $\geq 3$, the eventual goal is to find some $\tilde{r}, 0<\tilde{r} \leq r$, and a holomorphic map $\vec{p}: \Delta_{\tilde{r}} \rightarrow \mathbb{C}^{n}$, so that the transformation $\tilde{z}=\vec{z}+\vec{p}(\vec{z})$ is a biholomorphism with domain $\Delta_{\tilde{r}}$ taking $M$ to $\tilde{M}$. That is, if $\vec{z} \in M \cap \Delta_{\tilde{r}}$, then $\tilde{z}$ satisfies (6). However, in this section we are only looking for $\vec{p}$ that is a solution of (10)-(11).

Some steps of the proof of Theorem 5.6 below will decompose series into subseries and their complex conjugates, where these preliminary lemmas on the $|e|_{r}$ norm will be useful.

Lemma 5.3. Given $0<R<r$ and complex coefficients $a_{j k I}, b_{j k I}$, if

$$
\left|\sum a_{j k I} z_{1}^{j} \zeta^{k} x^{I}\right|_{r} \leq K
$$

and for complex $x$ with $\left|x_{s}\right|<r, j, k=0,1,2,3, \ldots$,

$$
\left|\sum_{I} b_{j k I} x^{I}\right| \leq\left|\sum_{I} a_{j k I} x^{I}\right|
$$

then

$$
\left|\sum b_{j k I} z_{1}^{j} \zeta^{k} x^{I}\right|_{R} \leq \frac{K r^{2}}{(r-R)^{2}} .
$$

Proof. For $\left(z_{1}, \zeta, x\right) \in D_{r}$, these series are absolutely convergent and equal:

$$
\sum a_{j k I} z_{1}^{j} \zeta^{k} x^{I}=\sum_{j=0}^{\infty}\left(\sum_{k=0}^{\infty}\left(\sum_{I} a_{j k I} x^{I}\right) \zeta^{k}\right) z_{1}^{j} .
$$

Using Cauchy's estimate [Ahlfors 1979] twice, we obtain

$$
\left|\sum_{k=0}^{\infty}\left(\sum_{I} a_{j k I} x^{I}\right) \zeta^{k}\right| \leq \frac{K}{r^{j}} \quad \text { and } \quad\left|\sum_{I} a_{j k I} x^{I}\right| \leq \frac{K}{r^{k} r^{j}}
$$


For $\left(z_{1}, \zeta, x\right) \in D_{r}$, the series $\sum b_{j k I} z_{1}^{j} \zeta^{k} x^{I}$ is absolutely convergent, and for $\left(z_{1}, \zeta, x\right) \in D_{R}$ :

$$
\begin{aligned}
\left|\sum b_{j k I} z_{1}^{j} \zeta^{k} x^{I}\right| & =\left|\sum_{j=0}^{\infty}\left(\sum_{k=0}^{\infty}\left(\sum_{I} b_{j k I} x^{I}\right) \zeta^{k}\right) z_{1}^{j}\right| \\
& \leq \sum_{j=0}^{\infty}\left(\sum_{k=0}^{\infty}\left|\sum_{I} b_{j k I} x^{I}\right||\zeta|^{k}\right)\left|z_{1}\right|^{j} \\
& \leq \sum_{j=0}^{\infty}\left(\left.\sum_{k=0}^{\infty}\left|\sum_{I} a_{j k I} x^{I}\right| \zeta\right|^{k}\right)\left|z_{1}\right|^{j} \\
& \leq \sum_{j=0}^{\infty}\left(\sum_{k=0}^{\infty} \frac{K}{r^{k} r^{j}}|\zeta|^{k}\right)\left|z_{1}\right|^{j}=\sum_{j, k} K\left(\frac{|\zeta|}{r}\right)^{k}\left(\frac{\left|z_{1}\right|}{r}\right)^{j} \\
& =K \frac{1}{1-|\zeta| / r} \frac{1}{1-\left|z_{1}\right| / r}=\frac{K r^{2}}{(r-|\zeta|)\left(r-\left|z_{1}\right|\right)}<\frac{K r^{2}}{(r-R)^{2}}
\end{aligned}
$$

In the applications of the lemma, for each pair $(j, k)$, the coefficients $b_{j k I}$ will either be zero for all $I$ or equal to $a_{j k I}$ for all $I$, so the estimate in the hypothesis is satisfied.

Notation 5.4. On the complex vector space of formal power series, define the real structure operator

$$
e=\sum e^{a b I} z_{1}^{a} \zeta^{b} x^{I} \mapsto e^{\prime}=\sum \overline{e^{a b I}} \zeta^{a} z_{1}^{b} x^{I} .
$$

Lemma 5.5. For $r>0$, the restriction of the map (12) to the subspace $\left\{e:|e|_{r}<\infty\right\}$ is an isometry.

Proof. The equality of norms uses a change of variables that does not change the radius length $r$.

$$
\begin{aligned}
\left|e^{\prime}\right|_{r} & =\sup _{\left(z_{1}, \zeta, x\right) \in D_{r}}\left|\sum \overline{e^{a b I}} \zeta^{a} z_{1}^{b} x^{I}\right|=\sup _{\left(\zeta^{\prime}, z_{1}^{\prime}, x^{\prime}\right)=(\bar{z}, \bar{\zeta}, \bar{x}) \in D_{r}}\left|\sum \overline{e^{a b I}} \overline{z_{1}^{\prime}} \overline{\zeta^{\prime}} \overline{x^{\prime}}\right| \\
& =\sup _{\left(\zeta^{\prime}, z_{1}^{\prime}, x^{\prime}\right) \in D_{r}}\left|\overline{\sum e^{a b I}\left(z_{1}^{\prime}\right)^{a}\left(\zeta^{\prime}\right)^{b}\left(x^{\prime}\right)^{I}}\right|=\sup _{\left(z_{1}^{\prime}, \zeta^{\prime}, x^{\prime}\right) \in D_{r}}\left|\sum e^{a b I}\left(z_{1}^{\prime}\right)^{a}\left(\zeta^{\prime}\right)^{b}\left(x^{\prime}\right)^{I}\right| \\
& =|e|_{r}
\end{aligned}
$$

Of course, this map is a representation of complex conjugation: given a series $e\left(z_{1}, \bar{z}_{1}, x\right)$ for real $x$, which "complexifies" to $e=e\left(z_{1}, \zeta, x\right)$ for $\left(z_{1}, \zeta, x\right) \in D_{r}$ for the purposes of finding its norm as in Notation 5.2, expanding $\overline{e\left(z_{1}, \bar{z}_{1}, x\right)}$ as a series in $\left(z_{1}, \bar{z}_{1}, x\right)$ and then complexifying gives $e^{\prime}=e^{\prime}\left(z_{1}, \zeta, x\right)$. 
In an attempt to simplify the notation by avoiding an excess of indices in an already intricate calculation, the following theorem will focus on one particular dimension pair $(m, n)$. In order to represent the most general behavior, we want $m<n-1$, so there is a $z_{t}$ equation in (5), and also $m>\frac{2}{3}(n+1)$, so there is a variable $x_{m-1}$ that does not appear in the quadratic part of the defining equations. The smallest pair where both conditions occur is $m=7, n=9$, so we will be considering a real 7 -manifold in $\mathbb{C}^{9}$, where the coordinates of the tangent plane are $z_{1}, x_{2}, \ldots, x_{6}$, and the CR singular locus in $M$ near $\overrightarrow{0}$ is a real curve tangent to the $x_{6}$ axis at the origin.

Theorem 5.6. Given $r>0$ and $\vec{e}\left(z_{1}, \zeta, x\right)$ convergent on $D_{r}$ with $|\vec{e}|_{r}<\infty$ and degree $d \geq 3$, there exists $\vec{p}$ that is convergent on $\Delta_{r}$ and satisfies these properties:

(a) $\vec{p}$ solves the following case of the system of equations (10)-(11):

$$
\begin{aligned}
0 & =E_{s}\left(z_{1}, \bar{z}_{1}, x\right)+\operatorname{Im} p_{s}(\vec{z}) \quad \text { for } s=2, \ldots, 6, \\
0 & =e_{7}\left(z_{1}, \bar{z}_{1}, x\right)+p_{7}(\vec{z})-\overline{p_{1}(\vec{z})}\left(x_{4}+i x_{5}\right)-\bar{z}_{1}\left(\operatorname{Re} p_{4}(\vec{z})+i \operatorname{Re} p_{5}(\vec{z})\right), \\
0 & =e_{8}\left(z_{1}, \bar{z}_{1}, x\right)+p_{8}(\vec{z})-2 \bar{z}_{1} \overline{p_{1}(\vec{z})}, \\
0 & =e_{9}\left(z_{1}, \bar{z}_{1}, x\right)+p_{9}(\vec{z}) \\
& \quad-\bar{z}_{1}\left(p_{1}(\vec{z})+\operatorname{Re} p_{2}(\vec{z})+i \operatorname{Re} p_{3}(\vec{z})\right)-\overline{\left(p_{1}(\vec{z})\right)}\left(z_{1}+x_{2}+i x_{3}\right),
\end{aligned}
$$

where

$$
\vec{z}=\left(z_{1}, x_{2}, x_{3}, x_{4}, x_{5}, x_{6}, \bar{z}_{1}\left(x_{4}+i x_{5}\right), \bar{z}_{1}^{2}, \bar{z}_{1}\left(z_{1}+x_{2}+i x_{3}\right)\right) .
$$

(b) $\left\|p_{1}\right\|_{r} \leq 3\left|e_{8}\right|_{r} /(2 r),\left\|p_{8}\right\|_{r} \leq 4\left|e_{8}\right|_{r}$ and, for any $0<R<r$,

$$
\begin{aligned}
& \left\|p_{2}\right\|_{R} \leq \frac{3\left|e_{9}\right|_{r}+18\left|e_{8}\right|_{r}}{R}+\left(\frac{8 r^{2}}{(r-R)^{2}}+10\right)\left|E_{2}\right|_{r}+\left(\frac{4 r^{2}}{(r-R)^{2}}+4\right)\left|E_{3}\right|_{r}, \\
& \left\|p_{3}\right\|_{R} \leq \frac{3\left|e_{9}\right|_{r}+18\left|e_{8}\right|_{r}}{R}+\left(\frac{4 r^{2}}{(r-R)^{2}}+4\right)\left|E_{2}\right|_{r}+\left(\frac{8 r^{2}}{(r-R)^{2}}+10\right)\left|E_{3}\right|_{r}, \\
& \left\|p_{4}\right\|_{R} \leq \frac{3\left|e_{7}\right|_{r}+9\left|e_{8}\right|_{r}}{R}+\left(\frac{8 r^{2}}{(r-R)^{2}}+10\right)\left|E_{4}\right|_{r}+\left(\frac{4 r^{2}}{(r-R)^{2}}+4\right)\left|E_{5}\right|_{r} \\
& \left\|p_{5}\right\|_{R} \leq \frac{3\left|e_{7}\right|_{r}+9\left|e_{8}\right|_{r}}{R}+\left(\frac{4 r^{2}}{(r-R)^{2}}+4\right)\left|E_{4}\right|_{r}+\left(\frac{8 r^{2}}{(r-R)^{2}}+10\right)\left|E_{5}\right|_{r} \\
& \left\|p_{6}\right\|_{R} \leq \frac{20 r^{2}}{(r-R)^{2}}\left|E_{6}\right|_{r}, \\
& \left\|p_{7}\right\|_{R} \leq 4\left|e_{7}\right|_{r}+12\left|e_{8}\right|_{r}+8 R\left(\frac{r^{2}}{(r-R)^{2}}+1\right)\left(\left|E_{4}\right|_{r}+\left|E_{5}\right|_{r}\right), \\
& \left\|p_{9}\right\|_{R} \leq 4\left|e_{9}\right|_{r}+24\left|e_{8}\right|_{r}+8 R\left(\frac{r^{2}}{(r-R)^{2}}+1\right)\left(\left|E_{2}\right|_{r}+\left|E_{3}\right|_{r}\right) .
\end{aligned}
$$


Proof. First, notice that if $\vec{p}\left(z_{1}, \ldots, z_{9}\right)$ is a formal series solution of (13)-(14), it does not follow that $\vec{p}$ is convergent at any point (other than the origin). For example, with any component $p_{j}$, the series expressions $p_{j}(\vec{z})$ and

$$
p_{j}(\vec{z})+\left(\left(z_{1}+z_{2}+i z_{3}\right)^{2} z_{8}-z_{9}^{2}\right) \cdot \mathrm{Q}(\vec{z})
$$

are formally the same when restricted to $\vec{z}$ as in (14), for any (possibly divergent) series Q. So, if one formal solution $\vec{p}$ exists, then there exist infinitely many divergent solutions. There may also exist formal series solutions that are convergent only on some neighborhood of the origin much smaller than that claimed in the theorem.

Continuing with the abbreviation $x=x_{2}, x_{3}, x_{4}, x_{5}, x_{6}$, and also using $z=$ $z_{2}, z_{3}, z_{4}, z_{5}, z_{6}$, the following choice of normalization will simplify the construction of the solution $\vec{p}$ satisfying the claimed convergence and bounds:

$$
p_{1}(\vec{z})=p_{1}\left(z_{1}, z, z_{8}\right), \quad p_{j}(\vec{z})=p_{j}^{E}\left(z_{1}, z, z_{8}\right)+z_{9} p_{j}^{O}\left(z_{1}, z, z_{8}\right),
$$

for $j=2, \ldots, 9$. Note that $\vec{p}$ does not depend on $z_{7}$, and the first component $p_{1}$ does not depend on $z_{9}$. We may make the further assumption that $p_{1}$ is an even function of $z_{1}: p_{1}\left(z_{1}, z, z_{8}\right)=p_{1}\left(-z_{1}, z, z_{8}\right)$. The remaining components, $p_{j}$, have some terms not depending on $z_{9}$, labeled $p_{j}^{E}$, and other terms which have exactly one linear factor of $z_{9}$. The $p_{j}^{E}$ and $p_{j}^{O}$ terminology corresponds to even and odd powers of $\bar{z}_{1}$ which appear after the substitution of (14) into $\vec{p}$. The choice that $\vec{p}$ has at most linear terms in $z_{9}=\bar{z}_{1}\left(z_{1}+x_{2}+i x_{3}\right)$ is made to avoid high powers of the nonmonomial quantity $\bar{z}_{1}\left(z_{1}+x_{2}+i x_{3}\right)$, since as in [Coffman 1997], any multinomial coefficients in the series expansion of $\vec{p}(\vec{z})$ could be large enough to affect the size of the domain of convergence.

We begin with the $e_{8}$ equation of the system (13). If the series expansion of $e_{8}$ had only even powers of $\bar{z}_{1}$, then it would be a very simple matter to compare the coefficients of $e_{8}\left(z_{1}, \bar{z}_{1}, x\right)$ and $p_{8}^{E}\left(z_{1}, x, \bar{z}_{1}^{2}\right)$, and get a solution of the equation with $p_{8}^{O}=p_{1}=0$. The odd powers of $\bar{z}_{1}$ in $e_{8}$ make the $p_{8}^{O}$ and $p_{1}$ quantities necessary to solve the equation. The consideration of the terms of the components of the given quantity $\vec{e}$ which are even or odd in $\bar{z}_{1}$ was part of the analysis of [Beloshapka 1997] and [Coffman 1997] of the formal normal form problem, and even/odd decompositions also appeared in analogous calculations in [Whitney 1943]. However, to deal with the nonmonomial property of the quadratic normal form, there will be some rearrangements of the terms in the series which were not required in Whitney's work. First, decompose $e_{8}$ into even and odd parts $e_{8 A}, e_{8 B}$, $e_{8 C}$, and then apply an add-and-subtract trick to $e_{8 C}$, as follows:

$$
\begin{aligned}
e_{8} & =\sum e_{8}^{a b I} z_{1}^{a} \bar{z}_{1}^{b} x^{I}=e_{8 A}+e_{8 B}+e_{8 C}, \\
e_{8 A} & =\sum_{b \text { even }} e_{8}^{a b I} z_{1}^{a} \bar{z}_{1}^{b} x^{I},
\end{aligned}
$$




$$
\begin{aligned}
& e_{8 B}=\sum_{a \text { even, } b \text { odd }} e_{8}^{a b I} z_{1}^{a} \bar{z}_{1}^{b} x^{I}, \\
& e_{8 C}=\sum_{a, b \text { odd }} e_{8}^{a b I} z_{1}^{a} \bar{z}_{1}^{b} x^{I}=e_{8 D}+e_{8 E}, \\
& e_{8 D}=\bar{z}_{1}\left(z_{1}+x_{2}+i x_{3}\right) \sum_{a, b \text { odd }} e_{8}^{a b I} z_{1}^{a-1} \bar{z}_{1}^{b-1} x^{I}, \\
& e_{8 E}=-\left(x_{2}+i x_{3}\right) \sum_{a, b \text { odd }} e_{8}^{a b I} z_{1}^{a-1} \bar{z}_{1}^{b} x^{I} .
\end{aligned}
$$

Let $f_{8}=e_{8 B}+e_{8 E}$, so $f_{8}\left(z_{1}, \bar{z}_{1}, x\right)$ is even in $z_{1}$ and odd in $\bar{z}_{1}$. Then, combining $e_{8}=e_{8 A}+e_{8 D}+f_{8}$ with the normalization for $p_{1}$ and $p_{8}$ in the $e_{8}$ equation from (13), a straightforward (by construction) comparison of coefficients yields

$$
\begin{aligned}
& 0=e_{8 A}\left(z_{1}, \bar{z}_{1}, x\right)+p_{8}^{E}\left(z_{1}, x, \bar{z}_{1}^{2}\right), \\
& 0=e_{8 D}\left(z_{1}, \bar{z}_{1}, x\right)+\bar{z}_{1}\left(z_{1}+x_{2}+i x_{3}\right) p_{8}^{O}\left(z_{1}, x, \bar{z}_{1}^{2}\right), \\
& 0=f_{8}\left(z_{1}, \bar{z}_{1}, x\right)-2 \bar{z}_{1} \overline{p_{1}\left(z_{1}, x, \bar{z}_{1}^{2}\right)} .
\end{aligned}
$$

If $p_{8}^{E}\left(z_{1}, z, z_{8}\right)=\sum p_{8}^{a c I} z_{1}^{a} z_{8}^{c} z^{I}$, then the coefficient $p_{8}^{a c I}$ must be equal to $-e_{8}^{a, 2 c, I}$, and we get an estimate for the norm of $p_{8}^{E}$ on the polydisc $\Delta_{r} \subseteq \mathbb{C}^{9}$ :

$$
\begin{aligned}
\left\|p_{8}^{E}\right\|_{r} & =\sup _{\vec{z} \in \Delta_{r}}\left|p_{8}^{E}(\vec{z})\right|=\sup _{\substack{\left|z_{1}\right|<r,\left|x_{s}\right|<r,\left|\zeta^{2}\right|<r^{2}}}\left|p_{8}^{E}\left(z_{1}, x, \zeta^{2}\right)\right|=\sup _{\left(z_{1}, \zeta, x\right) \in D_{r}}\left|-e_{8 A}\left(z_{1}, \zeta, x\right)\right| \\
& =\left|e_{8}\right|_{r}=\left|\frac{1}{2}\left(e_{8}\left(z_{1}, \zeta, x\right)+e_{8}\left(z_{1},-\zeta, x\right)\right)\right|_{r} \leq\left|e_{8}\right|_{r} .
\end{aligned}
$$

By using the averaging formula to extract the even part of $e_{8}$, we can just apply the triangle inequality to get the estimate for the subseries instead of Lemma 5.3. There is a similar estimate for the other component $p_{8}^{O}$, but this time the Schwarz Lemma [Ahlfors 1979] is used in two steps:

$$
\begin{aligned}
\left\|z_{9} p_{8}^{O}\right\|_{r} & \leq\|z 9\|_{r}\left\|p_{8}^{O}\right\|_{r}=3 r^{2} \sup _{\substack{\left|z_{1}\right|<r,\left|x_{s}\right|<r,\left|\zeta^{2}\right|<r^{2}}}\left|p_{8}^{O}\left(z_{1}, x, \zeta^{2}\right)\right| \\
& =3 r^{2} \sup _{\left(z_{1}, \zeta, x\right) \in D_{r}}\left|\frac{-e_{8 D}\left(z_{1}, \zeta, x\right)}{\zeta\left(z_{1}+x_{2}+i x_{3}\right)}\right|=3 r^{2} \sup _{\left(z_{1}, \zeta, x\right) \in D_{r}^{*}}\left|\frac{-e_{8 C}\left(z_{1}, \zeta, x\right) \mid}{z_{1} \zeta}\right| \\
& \leq 3 r^{2} \sup _{\left(z_{1}, \zeta, x\right) \in D_{r}^{*}} \frac{\left(\left|z_{1}\right| / r\right) \sup _{\left|z_{1}\right|<r}\left|e_{8 C}\right|}{\left|z_{1}\right||\zeta|} \\
& \leq 3 r \sup _{\left(z_{1}, \zeta, x\right) \in D_{r}^{*}} \frac{\sup _{\left|z_{1}\right|<r}\left|(|\zeta| / r) \sup _{|\zeta|<r}\right| e_{8 C}||}{|\zeta|}=3\left|e_{8 C}\right|_{r} \\
& =\frac{3}{4}\left|e_{8}\left(z_{1}, \zeta, x\right)-e_{8}\left(z_{1},-\zeta, x\right)-e_{8}\left(-z_{1}, \zeta, x\right)+e_{8}\left(-z_{1},-\zeta, x\right)\right|_{r} \\
& \leq 3\left|e_{8}\right|_{r} .
\end{aligned}
$$

In some of these steps, we restricted to the open subset

$$
D_{r}^{*}=D_{r} \backslash\left(\left\{z_{1}=0\right\} \cup\{\zeta=0\}\right)
$$


which avoids division by 0 but, by the maximum principle, does not affect the supremum.

From $f_{8}=e_{8 B}+e_{8 E}$ and the Schwarz Lemma,

$$
\begin{aligned}
\left|f_{8}\right|_{r} & \leq\left|e_{8 B}\right|_{r}+\left|e_{8 E}\right|_{r} \\
& =\frac{1}{4}\left|e_{8}\left(z_{1}, \zeta, x\right)-e_{8}\left(z_{1},-\zeta, x\right)+e_{8}\left(-z_{1}, \zeta, x\right)-e_{8}\left(-z_{1},-\zeta, x\right)\right|_{r} \\
& \leq\left|e_{8}\right|_{r}+\left|x_{2}+i x_{3}\right|_{r} \cdot \frac{1}{r}\left|e_{8 C}\right|_{r} \leq 3\left|e_{8}\right|_{r} .
\end{aligned}
$$

Solving for $p_{1}$ involves complex conjugation, so we take care to work out a few steps. By comparing the coefficients of $f_{8}$ and $p_{1}$, we see that if $p_{1}\left(z_{1}, z, z_{8}\right)=$ $\sum_{\alpha \text { even }} p_{1}^{\alpha \beta I} z_{1}^{\alpha} z_{8}^{\beta} z^{I}$, then $p_{1}^{\alpha \beta I}=\frac{1}{2} \overline{f_{8}^{2 \beta, \alpha+1, I}}$. Using the Schwarz Lemma and Lemma 5.5, we obtain

$$
\begin{aligned}
\left\|p_{1}\right\|_{r} & =\sup _{\left(z_{1}, \zeta, x\right) \in D_{r}}\left|\sum_{\alpha \text { even }} p_{1}^{\alpha \beta I} z_{1}^{\alpha} \zeta^{2 \beta} x^{I}\right|=\sup _{\left(z_{1}, \zeta, x\right) \in D_{r}}\left|\sum_{\alpha \text { even }} \frac{1}{2} \overline{f_{8}^{2 \beta, \alpha+1, I}} z_{1}^{\alpha} \zeta^{2 \beta} x^{I}\right| \\
& =\sup _{\left(z_{1}, \zeta, x\right) \in D_{r}}\left|\sum_{a \text { even, } b \text { odd }} \frac{\overline{f_{8}^{a b I}} \zeta^{a} z_{1}^{b} x^{I}}{2 z_{1}}\right|=\left|\frac{f_{8}^{\prime}\left(z_{1}, \zeta, x\right)}{2 z_{1}}\right|_{r} \\
& \leq \frac{1}{2 r}\left|f_{8}^{\prime}\right|_{r}=\frac{1}{2 r}\left|f_{8}\right|_{r} \leq \frac{3}{2 r}\left|e_{8}\right|_{r} .
\end{aligned}
$$

By construction, $p_{1}$ has weight $d-1$ and $p_{8}$ has weight $d$.

Moving next to the $E_{6}$ equation of (13), split the real valued series $E_{6}$ into subseries, some real and some in complex conjugate pairs:

$$
\begin{aligned}
E_{6} & =e_{6 A}+\overline{e_{6 A}}+E_{6 B}+e_{6 C}+\overline{e_{6 C}}+e_{6 D}+\overline{e_{6 D}}+E_{6 E}, \\
e_{6 A} & =\sum_{a>b, b \text { even }} E_{6}^{a b I} z_{1}^{a} \bar{z}_{1}^{b} x^{I}, \\
E_{6 B} & =\sum_{a \text { even }} E_{6}^{a a I} z_{1}^{a} \bar{z}_{1}^{a} x^{I}, \\
e_{6 C} & =\sum_{a>b, a \text { even, } b \text { odd }} E_{6}^{a b I} z_{1}^{a} \bar{z}_{1}^{b} x^{I}, \\
e_{6 D} & =\sum_{a>b, a, b \text { odd }} E_{6}^{a b I} z_{1}^{a} \bar{z}_{1}^{b} x^{I}, \\
E_{6 E} & =\sum_{a \text { odd }} E_{6}^{a a I} z_{1}^{a} \bar{z}_{1}^{a} x^{I} .
\end{aligned}
$$

By Lemma 5.3, we have

$$
\left|e_{6 A}\right|_{R} \leq \frac{r^{2}}{(r-R)^{2}}\left|E_{6}\right|_{r},
$$


and all the other subseries have the same bound. We rearrange two of these subseries to be able to compare coefficients with $p_{6}$ :

$$
\begin{aligned}
& e_{6 D}=e_{6 F}+e_{6 G}, \\
& e_{6 F}=\left(z_{1}+x_{2}+i x_{3}\right) \bar{z}_{1} \sum_{a>b, a, b \text { odd }} E_{6}^{a b I} z_{1}^{a-1} \bar{z}_{1}^{b-1} x^{I}, \\
& e_{6 G}=-\left(x_{2}+i x_{3}\right) \sum_{a>b, a, b \text { odd }} E_{6}^{a b I} z_{1}^{a-1} \bar{z}_{1}^{b} x^{I}, \\
& E_{6 E}=e_{6 H}+\overline{e_{6 H}}+e_{6 I}+\overline{e_{6 I}}, \\
& e_{6 H}=\frac{1}{2}\left(z_{1}+x_{2}+i x_{3}\right) \bar{z}_{1} \sum_{a \text { odd }} E_{6}^{a a I} z_{1}^{a-1} \bar{z}_{1}^{a-1} x^{I}, \\
& e_{6 I}=-\frac{1}{2}\left(x_{2}+i x_{3}\right) \sum_{a \text { odd }} E_{6}^{a a I} z_{1}^{a-1} \bar{z}_{1}^{a} x^{I},
\end{aligned}
$$

and collect some of these subseries back together:

$$
\begin{aligned}
& f_{6 A}\left(z_{1}, \bar{z}_{1}, x\right)=e_{6 A}+\overline{e_{6 I}}=\sum_{a>b, b \text { even }} f_{6 A}^{a b I} z_{1}^{a} \bar{z}_{1}^{b} x^{I}, \\
& f_{6 C}\left(z_{1}, \bar{z}_{1}, x\right)=\overline{e_{6 C}}+\overline{e_{6 G}}=\sum_{a<b, a \text { odd, } b \text { even }} f_{6 C}^{a b I} z_{1}^{a} \bar{z}_{1}^{b} x^{I},
\end{aligned}
$$

so

$$
E_{6}=f_{6 A}+\overline{f_{6 A}}+E_{6 B}+f_{6 C}+\overline{f_{6 C}}+e_{6 F}+\overline{e_{6 F}}+e_{6 H}+\overline{e_{6 H}}
$$

The unknown $p_{6}$ can also be expressed as a sum of subseries:

$$
\begin{aligned}
p_{6} & =p_{6}^{E}\left(z_{1}, z, z_{8}\right)+z_{9} p_{6}^{O}\left(z_{1}, z, z_{8}\right), \\
p_{6}^{E} & =p_{6 A}+p_{6 B}+p_{6 C}, \\
p_{6 A} & =\sum_{\alpha>2 \gamma} p_{6 A}^{\alpha \gamma I} z_{1}^{\alpha} z^{I} z_{8}^{\gamma}, \\
p_{6 B} & =\sum p_{6 B}^{\gamma I} z_{1}^{2 \gamma} z^{I} z_{8}^{\gamma}, \\
p_{6 C} & =\sum_{\alpha<2 \gamma, \alpha \text { odd }} p_{6 C}^{\alpha \gamma I} z_{1}^{\alpha} z^{I} z_{8}^{\gamma}, \\
p_{6}^{O} & =p_{6 D}+p_{6 E}, \\
p_{6 D} & =\sum_{\alpha>2 \gamma, \alpha \text { even }} p_{6 D}^{\alpha \gamma I} z_{1}^{\alpha} z^{I} z_{8}^{\gamma}, \\
p_{6 E} & =\sum p_{6 E}^{\gamma I} z_{1}^{2 \gamma} z^{I} z_{8}^{\gamma} .
\end{aligned}
$$


Comparing coefficients, the equation $0=E_{6}-\frac{1}{2} i\left(p_{6}-\overline{p_{6}}\right)$ from (13) turns into these five equations and their complex conjugates:

$$
\begin{aligned}
& 0=f_{6 A}-\frac{1}{2} i p_{6 A}, \\
& 0=\frac{1}{2} E_{6 B}-\frac{1}{2} i p_{6 B}, \\
& 0=f_{6 C}-\frac{1}{2} i p_{6 C}, \\
& 0=e_{6 F}-\frac{1}{2} i\left(z_{1}+x_{2}+i x_{3}\right) \bar{z}_{1} p_{6 D}, \\
& 0=e_{6 H}-\frac{1}{2} i\left(z_{1}+x_{2}+i x_{3}\right) \bar{z}_{1} p_{6 E} .
\end{aligned}
$$

Solving for each component of $p_{6}$ gives a weight $d$ quantity, and using Lemma 5.5, the Schwarz Lemma, and the previously mentioned estimates for the subseries of $E_{6}$, we get these estimates:

$$
\begin{aligned}
\left\|p_{6 A}\right\|_{R} & =\left|-2 i f_{6 A}\right|_{R}=2\left|e_{6 A}+e_{6 I}^{\prime}\right|_{R} \leq 2\left(\left|e_{6 A}\right|_{R}+\left|e_{6 I}\right|_{R}\right), \\
& \leq 2\left(\left|e_{6 A}\right|_{R}+\left|\frac{-\left(x_{2}+i x_{3}\right) E_{6 E}}{2 z_{1}}\right|_{R}\right), \\
& \leq 2\left(\frac{r^{2}\left|E_{6}\right|_{r}}{(r-R)^{2}}+\frac{2 R}{2} \cdot \frac{1}{R} \cdot \frac{r^{2}\left|E_{6}\right|_{r}}{(r-R)^{2}}\right)=\frac{4 r^{2}}{(r-R)^{2}}\left|E_{6}\right|_{r}, \\
\left\|p_{6 B}\right\|_{R} & =\left|-2 i \cdot \frac{1}{2} E_{6 B}\right|_{R} \leq \frac{r^{2}}{(r-R)^{2}}\left|E_{6}\right|_{r}, \\
\left\|p_{6 C}\right\|_{R} & =\left|-2 i f_{6 C}\right|_{R}=2\left|e_{6 C}^{\prime}+e_{6 G}^{\prime}\right|_{R}, \\
& \leq 2\left(\left|e_{6 C}\right|_{R}+\left|\frac{-\left(x_{2}+i x_{3}\right) e_{6 D}}{z_{1}}\right|_{R}\right), \\
& \leq 2\left(\frac{r^{2}\left|E_{6}\right|_{r}}{(r-R)^{2}}+2 R \cdot \frac{1}{R} \cdot \frac{r^{2}\left|E_{6}\right|_{r}}{(r-R)^{2}}\right)=\frac{6 r^{2}}{(r-R)^{2}}\left|E_{6}\right|_{r}, \\
\left\|z_{9} p_{6 D}\right\|_{R} & \leq\left\|z_{9}\right\|_{R}\left\|p_{6 D}\right\|_{R}=\left.\left.3 R^{2}\right|_{-2 i} \frac{e_{6 F}}{\left(z_{1}+x_{2}+i x_{3}\right) \zeta}\right|_{R}=6 R^{2}\left|\frac{e_{6 D}}{z_{1} \zeta}\right|_{R}, \\
& \leq 6\left|e_{6 D}\right|_{R} \leq \frac{6 r^{2}}{(r-R)^{2}}\left|E_{6}\right|_{r}, \\
\left\|z_{9} p_{6 E}\right\|_{R} & \leq 3 R^{2}\left|-2 i \frac{e_{6 H}}{\left(z_{1}+x_{2}+i x_{3}\right) \zeta}\right|_{R}=6 R^{2}\left|\frac{e_{6 E}}{2 z_{1} \zeta}\right|_{R} \leq \frac{3 r^{2}}{(r-R)^{2}}\left|E_{6}\right|_{r} .
\end{aligned}
$$

Finding $p_{2}, p_{3}, p_{4}, p_{5}$ is a bit trickier since each appears in more than one equation of (13). We will simultaneously solve for $p_{4}, p_{5}, p_{7}$, using a more involved comparison of coefficients, and similarly but independently, also $p_{2}, p_{3}, p_{9}$. 
To find $p_{4}, p_{5}$, and $p_{7}$, we consider the $E_{4}, E_{5}, e_{7}$ equations of (13), and use the previously found solution for $p_{1}$ to get the system with the unknowns on the left-hand side and the known $O(d)$ quantities on the right-hand side:

$$
\begin{aligned}
\operatorname{Im} p_{4} & =-E_{4}, \\
\operatorname{Im} p_{5} & =-E_{5}, \\
p_{7}-\bar{z}_{1} \operatorname{Re} p_{4}-i \bar{z}_{1} \operatorname{Re} p_{5} & =-e_{7}+\left(x_{4}+i x_{5}\right) \bar{p}_{1}
\end{aligned}
$$

Starting with the right-hand side of (16), the following decomposition of $E_{4}$ is different from that of $E_{6}$ :

$$
\begin{aligned}
E_{4} & =E_{4 A}+e_{4 B}+\overline{e_{4 B}}+e_{4 C}+\overline{e_{4 C}}+E_{4 D}, \\
E_{4 A} & =\sum_{a, b \text { even }} E_{4}^{a b I} z_{1}^{a} \bar{z}_{1}^{b} x^{I}, \\
e_{4 B} & =\sum_{a>b, a \text { odd, } b \text { even }} E_{4}^{a b I} z_{1}^{a} \bar{z}_{1}^{b} x^{I}, \\
e_{4 C} & =\sum_{a>b, a \text { even, } b \text { odd }} E_{4}^{a b I} z_{1}^{a} \bar{z}_{1}^{b} x^{I}, \\
E_{4 D} & =\sum_{a, b \text { odd }} E_{4}^{a b I} z_{1}^{a} \bar{z}_{1}^{b} x^{I}=e_{4 E}+e_{4 F}, \\
e_{4 E} & =\left(z_{1}+x_{2}+i x_{3}\right) \bar{z}_{1} \sum_{a, b \text { odd }} E_{4}^{a b I} z_{1}^{a-1} \bar{z}_{1}^{b-1} x^{I}, \\
e_{4 F} & =-\left(x_{2}+i x_{3}\right) \sum_{a, b \text { odd }} E_{4}^{a b I} z_{1}^{a-1} \bar{z}_{1}^{b} x^{I} .
\end{aligned}
$$

The $E_{4 A}$ piece is simply an even part, so $\left|E_{4 A}\right|_{r} \leq\left|E_{4}\right|_{r}$, and similarly for the odd part, $\left|E_{4 D}\right|_{r} \leq\left|E_{4}\right|_{r}$. The other two subseries satisfy the estimate from Lemma 5.3:

$$
\left|e_{4 B}\right|_{R} \leq \frac{r^{2}}{(r-R)^{2}}\left|E_{4}\right|_{r} \quad \text { and } \quad\left|e_{4 C}\right|_{R} \leq \frac{r^{2}}{(r-R)^{2}}\left|E_{4}\right|_{r} .
$$

We regroup some of these subseries:

$$
\begin{aligned}
m_{4}\left(z_{1}, \bar{z}_{1}, x\right) & =\overline{e_{4 B}}+e_{4 C}+e_{4 F}=\sum_{a \text { even, } b \text { odd }} m_{4}^{a b I} z_{1}^{a} \bar{z}_{1}^{b} x^{I}, \\
f_{4}\left(z_{1}, \bar{z}_{1}, x\right) & =E_{4 A}+e_{4 B}+\overline{e_{4 C}}+\overline{m_{4}}=\sum_{b \text { even }} f_{4}^{a b I} z_{1}^{a} \bar{z}_{1}^{b} x^{I} .
\end{aligned}
$$

Hence,

$$
E_{4}=f_{4}+e_{4 E}+m_{4}-\overline{m_{4}} .
$$

Similarly, $E_{5}=f_{5}+e_{5 E}+m_{5}-\overline{m_{5}}$, where $f_{5}$ is even in $\bar{z}_{1}$ and $m_{5}$ is even in $z_{1}$ and odd in $\bar{z}_{1}$. 
The estimates follow from Lemma 5.5 and the Schwarz Lemma:

$$
\begin{aligned}
\left|m_{4}\right|_{R} & =\left|e_{4 B}^{\prime}+e_{4 C}+e_{4 F}\right|_{R} \leq\left|e_{4 B}\right|_{R}+\left|e_{4 C}\right|_{R}+\left|e_{4 F}\right|_{R} \\
& \leq \frac{r^{2}}{(r-R)^{2}}\left|E_{4}\right|_{r}+\frac{r^{2}}{(r-R)^{2}}\left|E_{4}\right|_{r}+\left|x_{2}+i x_{3}\right|_{R}\left|\frac{E_{4 D}}{z_{1}}\right|_{R} \\
& \leq\left(\frac{2 r^{2}}{(r-R)^{2}}+2\right)\left|E_{4}\right|_{r}, \\
\left|f_{4}\right|_{R} & =\left|E_{4 A}+2 e_{4 B}+2 e_{4 C}^{\prime}+e_{4 F}^{\prime}\right|_{R} \leq\left(\frac{4 r^{2}}{(r-R)^{2}}+3\right)\left|E_{4}\right|_{r},
\end{aligned}
$$

and similarly for $m_{5}$ and $f_{5}$.

From the right-hand side of (18), let $f_{7}\left(z_{1}, \bar{z}_{1}, x\right)=-e_{7}+\left(x_{4}+i x_{5}\right) \overline{p_{1}\left(z_{1}, x, \bar{z}_{1}^{2}\right)}$, so

$$
\left|f_{7}\right|_{r} \leq\left|-e_{7}\right|_{r}+\left|\left(x_{4}+i x_{5}\right) \frac{f_{8}}{2 \zeta}\right|_{r} \leq\left|e_{7}\right|_{r}+3\left|e_{8}\right|_{r}
$$

It splits into even and odd parts, $f_{7}=f_{7 A}+f_{7 B}+f_{7 C}$, with

$$
\begin{aligned}
& f_{7 A}=\sum_{b \text { even }} f_{7 A}^{a b I} z_{1}^{a} \bar{z}_{1}^{b} x^{I} \\
& f_{7 B}=\sum_{a, b \text { odd }} f_{7 B}^{a b I} z_{1}^{a} \bar{z}_{1}^{b} x^{I}, \\
& f_{7 C}=\sum_{a \text { even, } b \text { odd }} f_{7 C}^{a b I} z_{1}^{a} \bar{z}_{1}^{b} x^{I},
\end{aligned}
$$

with $\left|f_{7 A}\right|_{r} \leq\left|f_{7}\right|_{r}$ and the same bound for $f_{7 B}, f_{7 C}$. Let

$$
\begin{aligned}
& g_{7 A}=f_{7 A}+i \bar{z}_{1} m_{4}-\bar{z}_{1} m_{5}=\sum_{b \text { even }} g_{7 A}^{a b I} z_{1}^{a} \bar{z}_{1}^{b} x^{I}, \\
& g_{7 B}=f_{7 B}-i \bar{z}_{1} \overline{m_{4}}+\bar{z}_{1} \overline{m_{5}}=\sum_{a, b \text { odd }} g_{7 B}^{a b I} z_{1}^{a} \bar{z}_{1}^{b} x^{I}=g_{7 C}+g_{7 D}, \\
& g_{7 C}=\left(z_{1}+x_{2}+i x_{3}\right) \bar{z}_{1} \sum_{a, b \text { odd }} g_{7 B}^{a b I} z_{1}^{a-1} \bar{z}_{1}^{b-1} x^{I}, \\
& g_{7 D}=-\left(x_{2}+i x_{3}\right) \sum_{a, b \text { odd }} g_{7 B}^{a b I} z_{1}^{a-1} \bar{z}_{1}^{b} x^{I} .
\end{aligned}
$$

Then

$$
f_{7}=g_{7 A}+g_{7 C}+g_{7 D}+f_{7 C}-\bar{z}_{1}\left(i m_{4}-i \overline{m_{4}}-m_{5}+\overline{m_{5}}\right)
$$

is in a form that compares to the left-hand side of (18) to give

$$
\begin{aligned}
p_{7}^{E} & =g_{7 A}, \\
\left(z_{1}+x_{2}+i x_{3}\right) \bar{z}_{1} p_{7}^{O} & =g_{7 C}, \\
-\bar{z}_{1}\left(\operatorname{Re} p_{4}+i \operatorname{Re} p_{5}\right) & =g_{7 D}+f_{7 C}-\bar{z}_{1}\left(i m_{4}-i \overline{m_{4}}-m_{5}+\overline{m_{5}}\right) .
\end{aligned}
$$


Equations (20) and (21) determine $p_{7}$, with the estimates

$$
\begin{aligned}
\left\|p_{7}^{E}\right\|_{R} & =\left|g_{7 A}\right|_{R}=\left|f_{7 A}+i \zeta m_{4}-\zeta m_{5}\right|_{R} \\
& \leq\left|f_{7}\right|_{r}+R\left(\frac{2 r^{2}}{(r-R)^{2}}+2\right)\left|E_{4}\right|_{r}+R\left(\frac{2 r^{2}}{(r-R)^{2}}+2\right)\left|E_{5}\right|_{r} \\
\left\|z_{9} p_{7}^{O}\right\|_{R} & \leq 3 R^{2}\left|\frac{g_{7 C}}{\left(z_{1}+x_{2}+i x_{3}\right) \zeta}\right|_{R}=3 R^{2}\left|\frac{g_{7 B}}{z_{1} \zeta}\right|_{R} \leq 3\left|f_{7 B}-i \zeta m_{4}^{\prime}+\zeta m_{5}^{\prime}\right|_{R} \\
& \leq 3\left|f_{7}\right|_{r}+3 R\left(\frac{2 r^{2}}{(r-R)^{2}}+2\right)\left|E_{4}\right|_{r}+3 R\left(\frac{2 r^{2}}{(r-R)^{2}}+2\right)\left|E_{5}\right|_{r} .
\end{aligned}
$$

Dividing (22) by $-\bar{z}_{1}$, then considering the real and imaginary parts and recalling (16) and (17), we get the system

$$
\begin{array}{ll}
\operatorname{Re} p_{4}=\operatorname{Re} \frac{g_{7 D}+f_{7 C}}{-\bar{z}_{1}}+i m_{4}-i \overline{m_{4}}, & \operatorname{Re} p_{5}=\operatorname{Im} \frac{g_{7 D}+f_{7 C}}{-\bar{z}_{1}}+i m_{5}-i \overline{m_{5}}, \\
\operatorname{Im} p_{4}=-E_{4}=-f_{4}-e_{4 E}-m_{4}+\overline{m_{4}}, & \operatorname{Im} p_{5}=-E_{5}=-f_{5}-e_{5 E}-m_{5}+\overline{m_{5}} .
\end{array}
$$

It is at this point that the second nondegeneracy condition - the full rank of the coefficient matrix (4) - is used: if the quadratic term $\epsilon_{7}^{5} \bar{z}_{1} x_{5}$ in $h_{7}$ had coefficient 0 instead of $i$, then $\operatorname{Re} p_{5}$ would not appear in the $e_{7}$ equality of (13), and (18) could not be solved this way.

Recombining the real and imaginary parts of $p_{4}, p_{5}$, there is (by construction) a convenient cancellation:

$$
\begin{aligned}
& p_{4}=\operatorname{Re} p_{4}+i \operatorname{Im} p_{4}=\operatorname{Re} \frac{g_{7 D}+f_{7 C}}{-\bar{z}_{1}}-i f_{4}-i e_{4 E}, \\
& p_{5}=\operatorname{Re} p_{5}+i \operatorname{Im} p_{5}=\operatorname{Im} \frac{g_{7 D}+f_{7 C}}{-\bar{z}_{1}}-i f_{5}-i e_{5 E} .
\end{aligned}
$$

These equations were set up so that $e_{4 E}$ and $e_{5 E}$ are the only terms on the righthand side with odd powers of $\bar{z}_{1}$, so $p_{4}=p_{4}^{E}+z_{9} p_{4}^{O}$ and $p_{5}=p_{5}^{E}+z_{9} p_{5}^{O}$ are each determined by a comparison of coefficients, and by construction, $p_{4}$ and $p_{5}$ have weight $d-1$ and satisfy the estimates

$$
\begin{aligned}
\left\|p_{4}^{E}\right\|_{R} & =\left|\frac{g_{7 D}}{-2 \zeta}+\frac{f_{7 C}}{-2 \zeta}+\frac{g_{7 D}^{\prime}}{-2 z_{1}}+\frac{f_{7 C}^{\prime}}{-2 z_{1}}-i f_{4}\right|_{R} \\
& \leq \frac{1}{R}\left|g_{7 D}\right|_{R}+\frac{1}{R}\left|f_{7 C}\right|_{R}+\left|f_{4}\right|_{R} \\
& \leq \frac{1}{R}\left|-\left(x_{2}+i x_{3}\right) \frac{g_{7 B}}{z_{1}}\right|_{R}+\frac{1}{R}\left|f_{7}\right|_{R}+\left|f_{4}\right|_{R} \\
& \leq \frac{3\left|e_{7}\right|_{r}+9\left|e_{8}\right|_{r}}{R}+\left(\frac{8 r^{2}}{(r-R)^{2}}+7\right)\left|E_{4}\right|_{r}+\left(\frac{4 r^{2}}{(r-R)^{2}}+4\right)\left|E_{5}\right|_{r}
\end{aligned}
$$




$$
\begin{aligned}
\left\|p_{5}^{E}\right\|_{R} & =\left|\frac{g_{7 D}}{-2 i \zeta}+\frac{f_{7 C}}{-2 i \zeta}-\frac{g_{7 D}^{\prime}}{-2 i z_{1}}-\frac{f_{7 C}^{\prime}}{-2 i z_{1}}-i f_{5}\right|_{R} \\
& \leq \frac{3\left|e_{7}\right|_{r}+9\left|e_{8}\right|_{r}}{R}+\left(\frac{4 r^{2}}{(r-R)^{2}}+4\right)\left|E_{4}\right|_{r}+\left(\frac{8 r^{2}}{(r-R)^{2}}+7\right)\left|E_{5}\right|_{r}, \\
\left\|z_{9} p_{4}^{O}\right\|_{R} & \leq\left\|z_{9} p_{4}^{O}\right\|_{r} \leq 3 r^{2}\left|\frac{-i e_{4 E}}{\left(z_{1}+x_{2}+i x_{3}\right) \zeta}\right|_{r}=3 r^{2}\left|\frac{E_{4 D}}{z_{1} \zeta}\right|_{r} \leq 3\left|E_{4}\right|_{r} \\
\left\|z_{9} p_{5}^{O}\right\|_{R} & \leq\left\|z_{9} p_{5}^{O}\right\|_{r} \leq 3 r^{2}\left|\frac{-i e_{5 E}}{\left(z_{1}+x_{2}+i x_{3}\right) \zeta}\right|_{r}=3 r^{2}\left|\frac{E_{5 D}}{z_{1} \zeta}\right|_{r} \leq 3\left|E_{5}\right|_{r} .
\end{aligned}
$$

The method of finding $p_{2}, p_{3}, p_{9}$ can be copied from the solution of $p_{4}, p_{5}, p_{7}$. In the place of (16), (17), (18), the system to be solved is

$$
\begin{aligned}
\operatorname{Im} p_{2} & =-E_{2}, \\
\operatorname{Im} p_{3} & =-E_{3}, \\
p_{9}-\bar{z}_{1} \operatorname{Re} p_{2}-i \bar{z}_{1} \operatorname{Re} p_{3} & =-e_{9}+\left(z_{1}+x_{2}+i x_{3}\right) \bar{p}_{1}+\bar{z}_{1} p_{1},
\end{aligned}
$$

and the right-hand side of the third equation can be abbreviated $f_{9}$, in analogy with $f_{7}$. The estimate (19) changes to

$$
\left|f_{9}\right|_{r} \leq\left|-e_{9}\right|_{r}+\left|\left(z_{1}+x_{2}+i x_{3}\right) \frac{f_{8}}{2 \zeta}\right|_{r}+\left|\zeta \frac{f_{8}^{\prime}}{2 z_{1}}\right|_{r} \leq\left|e_{9}\right|_{r}+6\left|e_{8}\right|_{r} .
$$

Both the construction of the solution and the estimates proceed by only changing the subscripts from 4,5,7 to 2,3,9, and adjusting the estimate for $f_{9}$ to get the claimed results - the second nondegeneracy condition on the quadratic part of $h_{9}$ is used here also in the same way.

Corollary 5.7. Given $\frac{2}{3}(n+1) \leq m<n, r>0$, and $\vec{e}\left(z_{1}, \zeta, x\right)$ convergent on $D_{r}$ with $|\vec{e}|_{r}<\infty$ and degree $d \geq 3$, there exists $\vec{p}$ that is convergent on $\Delta_{r}$, solves the system of equations (10)-(11), satisfies

$$
\left\|p_{1}\right\|_{r} \leq \frac{3}{2 r}\left|e_{n-1}\right|_{r} \quad \text { and } \quad\left\|p_{n-1}\right\|_{r} \leq 4\left|e_{n-1}\right|_{r},
$$

and, for any $0<R<r$, satisfies

$$
\begin{aligned}
& \left\|p_{2}\right\|_{R} \leq \frac{3\left|e_{n}\right|_{r}+18\left|e_{n-1}\right|_{r}}{R}+\left(\frac{8 r^{2}}{(r-R)^{2}}+10\right)\left|E_{2}\right|_{r}+\left(\frac{4 r^{2}}{(r-R)^{2}}+4\right)\left|E_{3}\right|_{r}, \\
& \left\|p_{3}\right\|_{R} \leq \frac{3\left|e_{n}\right|_{r}+18\left|e_{n-1}\right|_{r}}{R}+\left(\frac{4 r^{2}}{(r-R)^{2}}+4\right)\left|E_{2}\right|_{r}+\left(\frac{8 r^{2}}{(r-R)^{2}}+10\right)\left|E_{3}\right|_{r}, \\
& \left\|p_{n}\right\|_{R} \leq 4\left|e_{n}\right|_{r}+24\left|e_{n-1}\right|_{r}+8 R\left(\frac{r^{2}}{(r-R)^{2}}+1\right)\left(\left|E_{2}\right|_{r}+\left|E_{3}\right|_{r}\right) .
\end{aligned}
$$


Further, if $m>\frac{2}{3}(n+1)$, then

$$
\left\|p_{s}\right\|_{R} \leq \frac{20 r^{2}}{(r-R)^{2}}\left|E_{s}\right|_{r} \quad \text { for } s=2 n-2 m+2, \ldots, m-1,
$$

and if $m<n-1$, then for $t=m, \ldots, n-2$,

$$
\begin{aligned}
\left\|p_{2(t-m+2)}\right\|_{R} \leq \frac{3\left|e_{t}\right|_{r}+9\left|e_{n-1}\right|_{r}}{R}+\left(\frac{8 r^{2}}{(r-R)^{2}}\right. & +10)\left|E_{2(t-m+2)}\right|_{r} \\
& +\left(\frac{4 r^{2}}{(r-R)^{2}}+4\right)\left|E_{2(t-m+2)+1}\right|_{r},
\end{aligned}
$$

$$
\begin{aligned}
\left\|p_{2(t-m+2)+1}\right\|_{R} \leq \frac{3\left|e_{t}\right|_{r}+9\left|e_{n-1}\right|_{r}}{R}+\left(\frac{4 r^{2}}{(r-R)^{2}}+4\right)\left|E_{2(t-m+2)}\right|_{r} \\
+\left(\frac{8 r^{2}}{(r-R)^{2}}+10\right)\left|E_{2(t-m+2)+1}\right|_{r},
\end{aligned}
$$

$\left\|p_{t}\right\|_{R} \leq 4\left|e_{t}\right|_{r}+12\left|e_{n-1}\right|_{r}+8 R\left(\frac{r^{2}}{(r-R)^{2}}+1\right)\left(\left|E_{2(t-m+2)}\right|_{r}+\left|E_{2(t-m+2)+1}\right|_{r}\right)$.

Proof. The method of solution from the proof of Theorem 5.6 groups the system of equations into smaller subsystems that can be solved sequentially, so the generalization from $(7,9)$ to $(m, n)$ can be accomplished by a straightforward relabeling of subscripts (described below), resulting in similar estimates as claimed. The nondegeneracy conditions remain essential for any $(m, n)$.

The solution claimed by the corollary can be chosen to have the following form, where now $z$ abbreviates $z_{2}, \ldots, z_{m-1}$ and again $p_{1}$ is even in $z_{1}$ :

$$
\begin{aligned}
& p_{1}(\vec{z})=p_{1}\left(z_{1}, z, z_{n-1}\right) \\
& p_{j}(\vec{z})=p_{j}^{E}\left(z_{1}, z, z_{n-1}\right)+z_{n} p_{j}^{O}\left(z_{1}, z, z_{n-1}\right),
\end{aligned}
$$

for $j=2, \ldots, n$. For $m<n-1$, this $\vec{p}$ does not depend on $z_{m}, \ldots, z_{n-2}$.

The $e_{n-1}$ equation from (10) determines $p_{n-1}$ and $p_{1}$, exactly as in the solution of the $e_{8}$ equation, replacing the subscript 8 with $n-1$ in the first part of the preceding proof. The subscript 1 does not change.

If $m>\frac{2}{3}(n+1)$, then each of the $3 m-2 n-2$ individual $E_{s}$ equations, $s=$ $2 n-2 m+2, \ldots, m-1$, independently determines $p_{s}$, in analogy with the solution for $p_{6}$ in terms of $E_{6}$ in the second part of the preceding proof. If $m=\frac{2}{3}(n+1)$ (the case of an isolated singularity), there are no equations analogous to the proof's $E_{6}$ equation.

The subsystem of three equations determining $p_{2}, p_{3}, p_{n}$ in terms of $E_{2}, E_{3}$, $e_{n}$, and $p_{1}$, can be solved in analogy with the above $E_{2}, E_{3}, e_{9}$ group of equations (23), only the subscript 9 needs to change to $n$. If $m=n-1$, then those three equations are the only remaining ones in the system. 
If $m<n-1$, then there are $n-m-1$ more subsystems of three equations, to be solved for $p_{2(t-m+2)}, p_{2(t-m+2)+1}, p_{t}, t=m, \ldots, n-2$, in terms of $E_{2(t-m+2)}$, $E_{2(t-m+2)+1}, e_{t}$, and $p_{1}$, in analogy with equations (16)-(18). Solving each of these subsystems depends only on having solved for $p_{1}$, and not any other equations in the system (10).

It is not yet claimed that using the solution $\vec{p}$ of Theorem 5.6 or Corollary 5.7 in (2) defines a local biholomorphism; this will be shown later (Theorem 6.4), under certain conditions on $\vec{e}$ and $r$. The most important property so far of the solution $\vec{p}$ is that the norms of its components can be estimated on $\Delta_{R}$ for $R$ less than, but arbitrarily close to, $r$.

Corollary 5.8. Given $\frac{2}{3}(n+1) \leq m<n$, there is a constant $c_{1}>0$ (depending only on $m, n$ ) such that, for any $\vec{p}, \vec{e}$ as in Corollary 5.7 and any radius lengths $\rho, r$ with $\frac{1}{2}<\rho<r \leq 1$, we have

$$
\max _{j=1, \ldots, n}\left\{\left\|p_{j}\right\|_{\rho}\right\} \leq \frac{c_{1}|\vec{e}|_{r}}{(r-\rho)^{2}} \quad \text { and } \max _{j=1, \ldots, n}\left\{\sum_{k=1}^{n}\left\|\frac{d p_{k}}{d z_{j}}\right\|_{\rho}\right\} \leq \frac{c_{1}|\vec{e}|_{r}}{(r-\rho)^{3}} .
$$

Proof. Let $R=\frac{1}{2}(\rho+r)$. The bound on each $p_{j}$ follows from $\left\|p_{j}\right\|_{\rho} \leq\left\|p_{j}\right\|_{R}$ and the bounds from the previous corollary, using $\frac{1}{2}<R<r \leq 1$, and $16<$ $1 /(r-R)^{2}=4 /(r-\rho)^{2}$. The bounds for the derivatives of $p_{k}$ follow from this consequence of Cauchy's estimate (for which see [Ahlfors 1979]): If $0<R_{2}<R_{1}$ and $f(w)$ is holomorphic and bounded by $K$ for $|w|<R_{1}$, then $d f / d w$ is bounded by $K /\left(R_{1}-R_{2}\right)$ for $|w|<R_{2}$.

This fact can be applied with $K=\left\|p_{k}\right\|_{R}$ and $R_{1}-R_{2}=R-\rho=\frac{1}{2}(r-\rho)$ for the $z_{1}, \ldots, z_{m-1}$ derivatives, $R_{1}-R_{2}=R^{2}-\rho^{2}>R-\rho=\frac{1}{2}(r-\rho)$ for the $z_{n-1}$ derivatives, and $R_{1}-R_{2}=3 R^{2}-3 \rho^{2}>\frac{3}{2}(r-\rho)$ for the $z_{n}$ derivatives. The $z_{m}, \ldots, z_{n-2}$ derivatives are zero by construction.

The lower bound $r>\frac{1}{2}$ was important for the previous corollary, but it is not a significant $a$ priori restriction on the manifold $M$. By a real rescaling $\vec{z} \mapsto$ $\left(a_{1} z_{1}, \ldots, a_{1} z_{m-1}, a_{1}^{2} z_{m}, \ldots, a_{1}^{2} z_{n}\right)$, with $a_{1}>0$, equations (5) can be assumed to define $M$ for $\left|z_{1}\right|<1,\left|x_{s}\right|<1$; and for any $\eta>0$, there is a rescaling making $|\vec{e}|_{1}$ less than $\eta$.

\section{The new defining equations and some estimates}

To get a solution of the nonlinear equation (9) by iterating the solution of the linear equation, the rapid convergence technique will apply, closely following the methods used in [Moser 1985] on a different CR singularity problem. Each step along the way to a proof of Proposition 3.3 is stated as a theorem. 
Substituting the linear equation's normalized solution $\vec{p}$ from Corollary 5.7 into $E_{2}, \ldots, e_{n}$ in the right-hand side of the nonlinear equation (9) gives a quantity $\vec{q}$ depending on $z_{1}, \bar{z}_{1}, x$. Let

$$
\vec{z}=\left(z_{1}, x, \ldots, \bar{z}_{1}\left(x_{2(t-m+2)}+i x_{2(t-m+2)+1}\right), \ldots, \bar{z}_{1}^{2}, \bar{z}_{1}\left(z_{1}+x_{2}+i x_{3}\right)\right),
$$

as in (11), let

$$
\begin{aligned}
& \vec{z}+\vec{e}=\left(z_{1}, x_{2}+i E_{2}, \ldots, x_{m-1}+i E_{m-1}, \ldots,\right. \\
& \bar{z}_{1}\left(x_{2(t-m+2)}+i x_{2(t-m+2)+1}\right)+e_{t}, \ldots, \\
&\left.\bar{z}_{1}^{2}+e_{n-1}, \bar{z}_{1}\left(z_{1}+x_{2}+i x_{3}\right)+e_{n}\right),
\end{aligned}
$$

as in (8), and then define

$$
\vec{q}\left(z_{1}, \bar{z}_{1}, x\right)=\left(Q_{2}, \ldots, Q_{m-1}, q_{m}, \ldots, q_{n}\right)
$$

by

$$
\begin{aligned}
& Q_{s}=\operatorname{Im}\left(p_{s}(\vec{z}+\vec{e})-p_{s}(\vec{z})\right), \\
& q_{t}=p_{t}(\vec{z}+\vec{e})-p_{t}(\vec{z})-\left(x_{2(t-m+2)}+i x_{2(t-m+2)+1} \overline{\left(p_{1}(\vec{z}+\vec{e})-p_{1}(\vec{z})\right)}\right. \\
& -\bar{z}_{1} \cdot \operatorname{Re}\left(p_{2(t-m+2)}(\vec{z}+\vec{e})-p_{2(t-m+2)}(\vec{z})\right) \\
& -i \bar{z}_{1} \operatorname{Re}\left(p_{2(t-m+2)+1}(\vec{z}+\vec{e})-p_{2(t-m+2)+1}(\vec{z})\right) \\
& -\overline{p_{1}(\vec{z}+\vec{e})}\left(\operatorname{Re} p_{2(t-m+2)}(\vec{z}+\vec{e})+i \operatorname{Re} p_{2(t-m+2)+1}(\vec{z}+\vec{e})\right) \text {, } \\
& q_{n-1}=p_{n-1}(\vec{z}+\vec{e})-p_{n-1}(\vec{z})-2 \bar{z}_{1} \overline{\left(p_{1}(\vec{z}+\vec{e})-p_{1}(\vec{z})\right)}-{\overline{\left(p_{1}(\vec{z}+\vec{e})\right)}}^{2}, \\
& q_{n}=p_{n}(\vec{z}+\vec{e})-p_{n}(\vec{z})-\bar{z}_{1}\left(p_{1}(\vec{z}+\vec{e})-p_{1}(\vec{z})\right) \\
& -\bar{z}_{1}\left(\operatorname{Re}\left(p_{2}(\vec{z}+\vec{e})-p_{2}(\vec{z})\right)+i \operatorname{Re}\left(p_{3}(\vec{z}+\vec{e})-p_{3}(\vec{z})\right)\right) \\
& -\left(z_{1}+x_{2}+i x_{3}\right) \overline{\left(p_{1}(\vec{z}+\vec{e})-p_{1}(\vec{z})\right)} \\
& -\overline{\left(p_{1}(\vec{z}+\vec{e})\right)}\left(p_{1}(\vec{z}+\vec{e})+\operatorname{Re} p_{2}(\vec{z}+\vec{e})+i \operatorname{Re} p_{3}(\vec{z}+\vec{e})\right) \text {. }
\end{aligned}
$$

To outline the role of $\vec{q}$ in the argument, the next step (Theorem 6.2) will suppose that $\vec{p}\left(z_{1}, \ldots, z_{n}\right)$ is complex analytic on $\Delta_{\rho}$ and $|\vec{e}|_{\sigma}$ is small enough that $\vec{z} \in \Delta_{\sigma}$ implies $\vec{z}+\vec{e} \in \Delta_{\rho}$; hence $\vec{q}$ is a real analytic function for $\left(z_{1}, \bar{z}_{1}, x\right) \in D_{\sigma}$. If $\vec{q}\left(z_{1}, \bar{z}_{1}, x\right)$ happens to be identically zero, the manifold $M$ has been brought to normal form by the functions $\vec{p}$. Otherwise, the degree of $\vec{q}$ is at least $2 d-2$ by the construction of the solution $\vec{p}$, and defining $\vec{q}\left(z_{1}, \zeta, x\right)$ by Equations (26), with $\zeta$ formally substituted for $\bar{z}_{1}$ and allowing complex $x$, the norm $|\vec{q}|_{\sigma}$ can be bounded in terms of the norm of $\vec{e}$. Then later, in the proof of Theorem 6.6, converting $\vec{q}\left(z_{1}, \bar{z}_{1}, x\right)$ into an expression in $\tilde{z}_{1}, \overline{\tilde{z}}_{1}, \tilde{x}$ and equating it to the $\tilde{z}$ polynomial expression in (7) gives the defining equations of $M$ in the $\tilde{z}$ coordinate system. 
The case $\tilde{N}=N$ of the next result is [Coffman 2004, Lemma 4.1].

Lemma 6.1. Let $f=\left(f_{1}, \ldots, f_{\tilde{N}}\right): \mathbb{D}_{\boldsymbol{r}} \rightarrow \mathbb{C}^{\tilde{N}}$ be a holomorphic map with

$$
\max _{j=1, \ldots, N}\left\{\sum_{k=1}^{\tilde{N}} \sup _{\vec{z} \in \mathbb{D}_{r}}\left|\frac{d f_{k}}{d z_{j}}(\vec{z})\right|\right\} \leq K .
$$

Then, for $\vec{z}, \vec{z}^{\prime} \in \mathbb{D}_{\boldsymbol{r}}$,

$$
\sum_{k=1}^{\tilde{N}}\left|f_{k}\left(\vec{z}^{\prime}\right)-f_{k}(\vec{z})\right| \leq K \sum_{j=1}^{N}\left|z_{j}^{\prime}-z_{j}\right| .
$$

Theorem 6.2. There are some constants $c_{2}>0$ and $\delta_{1}>0$ (depending on $m, n$ ) such that if $\frac{1}{2}<\sigma<r \leq 1$, and $\vec{e}$ is as in Corollary 5.7, with $|\vec{e}|_{r} \leq \delta_{1}(r-\sigma)$, then

$$
|\vec{q}|_{\sigma} \leq \frac{c_{2}|\vec{e}|_{r}^{2}}{(r-\sigma)^{3}}
$$

Proof. Let $\rho=\frac{1}{2}(r+\sigma)$. Note that if $\delta_{1} \leq \frac{1}{2}$, the formal series for $\vec{q}$ is convergent on $D_{\sigma}$, since then, for $\left(z_{1}, \zeta, x\right) \in D_{r},\left|x_{s}+i E_{s}\right|<\sigma+\delta_{1}(r-\sigma) \leq \sigma+(\rho-\sigma)=\rho$, $\left|\zeta\left(x_{2(t-m+2)}+i x_{2(t-m+2)+1}\right)+e_{t}\right|<2 \sigma^{2}+(\rho-\sigma)<2 \sigma^{2}+(\rho-\sigma)(2(\rho+\sigma))=2 \rho^{2}$, and similarly $\left|\zeta^{2}+e_{n-1}\right|<\rho^{2}$ and $\left|\left(z_{1}+x_{2}+i x_{3}\right) \zeta+e_{n}\right|<3 \rho^{2}$, so $\vec{z}+\vec{e} \in \Delta_{\rho}$, which is contained in the domain of $\vec{p}$ by Corollary 5.7. The case $N=n, \tilde{N}=1$, $\mathbb{D}_{\boldsymbol{r}}=\Delta_{\rho}$ of Lemma 6.1 applies to $p_{k}: \Delta_{\rho} \rightarrow \mathbb{C}$, with

$$
\max _{j=1, \ldots, n}\left\{\left\|\frac{d p_{k}}{d z_{j}}\right\|_{\rho}\right\} \leq K=\frac{c_{1}|\vec{e}|_{r}}{(r-\rho)^{3}},
$$

by Corollary 5.8 , and $\vec{z}^{\prime}=\vec{z}+\vec{e} \in \Delta_{\rho}$, so the conclusion is

$$
\left|p_{k}(\vec{z}+\vec{e})-p_{k}(\vec{z})\right| \leq K\left(\left|E_{2}\right|_{r}+\cdots+\left|e_{n}\right|_{r}\right)=\frac{c_{1}|\vec{e}|_{r}}{(r-\rho)^{3}}|\vec{e}|_{r}=\frac{8 c_{1}|\vec{e}|_{r}^{2}}{(r-\sigma)^{3}} .
$$

This provides bounds for the differences that appear in (26), and the remaining terms are the products, where we can use $\frac{1}{2}<\sigma<\rho<r \leq 1$, the bound of Theorem 5.6 on the $p_{1}$ factor, and the bounds of Corollary 5.8 on the other factors. For example, for the $q_{t}$ equation of (26), in a case where $t=m<n-1$, part of the expression is the product

$$
\begin{aligned}
\sup _{D_{\sigma}} \mid & \left(p_{1}(\vec{z}+\vec{e})\right)^{\prime} \frac{p_{4}(\vec{z}+\vec{e})+\left(p_{4}(\vec{z}+\vec{e})\right)^{\prime}+i p_{5}(\vec{z}+\vec{e})+i\left(p_{5}(\vec{z}+\vec{e})\right)^{\prime}}{2} \mid \\
& \leq\left\|p_{1}\right\|_{\rho}\left(\left\|p_{4}\right\|_{\rho}+\left\|p_{5}\right\|_{\rho}\right) \leq \frac{3}{2 r}\left|e_{n-1}\right|_{r} \frac{2 c_{1}|\vec{e}|_{r}}{(r-\rho)^{2}}<\frac{6 c_{1}|\vec{e}|_{r}^{2}}{(r-\rho)^{2}}<\frac{12 c_{1}|\vec{e}|_{r}^{2}}{(r-\sigma)^{3}} .
\end{aligned}
$$


The following lemma on inverse functions will be used twice, in the construction of the new coordinate system and the new defining equations; a proof by a standard iteration procedure is sketched in [Coffman 2004].

Lemma 6.3. Suppose $0<R_{2, k}<R_{1, k}$ for $k=1, \ldots, N$, so that

$$
\mathbb{D}^{2}=\mathbb{D}_{\left(R_{2,1}, \ldots, R_{2, N}\right)} \subseteq \mathbb{D}^{1}=\mathbb{D}_{\left(R_{1,1}, \ldots, R_{1, N}\right)} .
$$

Let $f(\vec{z})=\left(f_{1}\left(z_{1}, \ldots, z_{N}\right), \ldots, f_{N}\left(z_{1}, \ldots, z_{N}\right)\right)$ be holomorphic on $\mathbb{D}^{1}$, with

and

$$
\max _{j=1, \ldots, N}\left\{\sum_{k=1}^{N} \sup _{\vec{z} \in \mathbb{D}^{1}}\left|\frac{d f_{k}}{d z_{j}}(\vec{z})\right|\right\} \leq K<1
$$

$$
\sum_{k=1}^{N} \sup _{\vec{z} \in \mathbb{D}^{2}}\left|f_{k}(\vec{z})\right| \leq(1-K) \min _{k=1, \ldots, N}\left\{R_{1, k}-R_{2, k}\right\} .
$$

Given $\vec{w} \in \mathbb{D}^{2}$, there exists a unique solution $\vec{z} \in \mathbb{D}^{1}$ of the equation

$$
\vec{w}=\vec{z}+f(\vec{z}),
$$

and this solution satisfies

$$
\sum_{k=1}^{N}\left|z_{k}-w_{k}\right| \leq \frac{1}{1-K} \sum_{k=1}^{N}\left|f_{k}(\vec{w})\right| .
$$

Theorem 6.4. There is some constant $\delta_{2}>0$ (depending on $m, n$ ) so that for any radius lengths $\frac{1}{2}<\sigma<r \leq 1$, and $\vec{e}, \vec{p}$ as in Corollary 5.7, with $|\vec{e}|_{r} \leq \delta_{2}(r-\sigma)^{3}$ and $\rho=\frac{1}{2}(r+\sigma)$, the transformation

$$
\Psi: \vec{z}=\left(z_{1}, \ldots, z_{n}\right) \mapsto \tilde{z}=\left(z_{1}+p_{1}(\vec{z}), \ldots, z_{n}+p_{n}(\vec{z})\right)
$$

has a holomorphic inverse $\psi(\tilde{z})=\vec{z}$ such that $\tilde{z} \in \Delta_{\sigma}$ implies $\psi(\tilde{z}) \in \Delta_{\rho}$.

Proof. By Corollary 5.8,

$$
\max _{j=1, \ldots, n}\left\{\sum_{k=1}^{n}\left\|\frac{d p_{k}}{d z_{j}}\right\|_{\rho}\right\} \leq \frac{c_{1}|\vec{e}|_{r}}{(r-\rho)^{3}} \leq \frac{c_{1} \delta_{2}(r-\sigma)^{3}}{(r-\rho)^{3}}=8 \delta_{2} c_{1} \leq \frac{1}{2}=K,
$$

if $\delta_{2} \leq 1 /\left(16 c_{1}\right)$. Also by Corollary 5.8 ,

$$
\sum_{k=1}^{n}\left\|p_{k}\right\|_{\sigma} \leq \frac{n c_{1}|\vec{e}|_{r}}{(r-\sigma)^{2}} \leq n c_{1} \delta_{2}(r-\sigma) \leq(1-K)(\rho-\sigma)
$$

if $\delta_{2} \leq 1 /\left(4 n c_{1}\right)$. The hypotheses of Lemma 6.3 are satisfied with $\Delta_{\sigma} \subseteq \Delta_{\rho}$, and $R_{1, k}-R_{2, k} \geq \rho-\sigma$, so given $\tilde{z} \in \Delta_{\sigma}$, there exists a unique $\vec{z} \in \Delta_{\rho}$ such that $\tilde{z}=\left(z_{1}+p_{1}(\vec{z}), \ldots, z_{n}+p_{n}(\vec{z})\right)$. This defines $\psi$ so that $\Psi \circ \psi$ is the identity map on $\Delta_{\sigma}$. 
For $\left(z_{1}, \zeta, x\right) \in D_{R_{1}} \subseteq \mathbb{C}^{m}$, define $z^{c} \in \mathbb{C}^{n}$ by

$$
\begin{aligned}
& z^{c}=\left(z_{1}, x_{2}+i E_{2}\left(z_{1}, \zeta, x\right),\right. \ldots, x_{m-1}+i E_{m-1}\left(z_{1}, \zeta, x\right), \ldots, \\
& \zeta\left(x_{2(t-m+2)}+\right.\left.i x_{2(t-m+2)+1}\right)+e_{t}\left(z_{1}, \zeta, x\right), \ldots, \\
&\left.\zeta^{2}+e_{n-1}\left(z_{1}, \zeta, x\right), \zeta\left(z_{1}+x_{2}+i x_{3}\right)+e_{n}\left(z_{1}, \zeta, x\right)\right)
\end{aligned}
$$

and define a map $\tau: D_{R_{1}} \rightarrow \mathbb{C}^{m}$ by

$$
\begin{aligned}
\tau\left(z_{1}, \zeta, x\right)= & \left(\tau_{1}\left(z_{1}, \zeta, x\right), \ldots, \tau_{m}\left(z_{1}, \zeta, x\right)\right) \\
= & \left(z_{1}+p_{1}\left(z^{c}\right), \zeta+\left(p_{1}\left(z^{c}\right)\right)^{\prime}, x_{2}+\frac{1}{2}\left(p_{2}\left(z^{c}\right)+\left(p_{2}\left(z^{c}\right)\right)^{\prime}\right), \ldots,\right. \\
& \left.x_{m-1}+\frac{1}{2}\left(p_{m-1}\left(z^{c}\right)+\left(p_{m-1}\left(z^{c}\right)\right)^{\prime}\right)\right) .
\end{aligned}
$$

Theorem 6.5. There is some constant $\delta_{3}>0$ (depending on $m, n$ ) so that for any radius lengths $\frac{1}{2}<r^{\prime}<r \leq 1$, with $\sigma=r^{\prime}+\frac{1}{3}\left(r-r^{\prime}\right)$, and any $\vec{e}, \vec{p}$ as in Corollary 5.7, with $|\vec{e}|_{r} \leq \delta_{3}\left(r-r^{\prime}\right)^{3}$, the transformation $\tau:\left(z_{1}, \zeta, x\right) \mapsto\left(\tilde{z}_{1}, \tilde{\zeta}, \tilde{x}\right)$ has a holomorphic inverse $\phi\left(\tilde{z}_{1}, \tilde{\zeta}, \tilde{x}\right)=\left(z_{1}, \zeta, x\right)$ such that if $\left(\tilde{z}_{1}, \tilde{\zeta}, \tilde{x}\right) \in D_{r^{\prime}}$, then $\phi\left(\tilde{z}_{1}, \tilde{\zeta}, \tilde{x}\right) \in D_{\sigma}$

Proof. Let $\rho=r^{\prime}+\frac{2}{3}\left(r-r^{\prime}\right)$, so $\sigma-r^{\prime}=\rho-\sigma=r-\rho=\frac{1}{3}\left(r-r^{\prime}\right)<\frac{1}{6}$, and let $\bar{r}=\frac{1}{2}\left(r+r^{\prime}\right)$, so $\frac{1}{2}<r^{\prime}<\sigma<\bar{r}<\rho<r \leq 1$. If $\left(z_{1}, \zeta, x\right) \in D_{\bar{r}}$, and $\delta_{3} \leq \frac{2}{3}$, then $\left|E_{2}\left(z_{1}, \zeta, x\right)\right| \leq \delta_{3}\left(r-r^{\prime}\right)^{3}=216 \delta_{3}(\rho-\bar{r})^{3}<\left(216 / 12^{2}\right) \delta_{3}(\rho-\bar{r}) \leq \rho-\bar{r}$, and similarly $\left|e_{n-1}\left(z_{1}, \zeta, x\right)\right|<\rho^{2}-\bar{r}^{2}$, etc., so $z^{c} \in \Delta_{\rho}$, and $\vec{p}\left(z^{c}\right)$ and $\tau$ are welldefined and holomorphic on $D_{\bar{r}}$. Using Cauchy's estimate as in Corollary 5.8, for $\left(z_{1}, \zeta, x\right) \in D_{\sigma}$,

$$
\left|\frac{d}{d z_{1}} p_{2}\left(z^{c}\right)\right| \leq \frac{\left|p_{2}\left(z^{c}\right)\right|_{\bar{r}}}{\bar{r}-\sigma} \leq \frac{\left\|p_{2}\right\|_{\rho}}{\frac{1}{2}(\rho-\sigma)} \leq \frac{2 c_{1}|\vec{e}|_{r}}{(\rho-\sigma)(r-\rho)^{2}}=\frac{54 c_{1}|\vec{e}|_{r}}{\left(r-r^{\prime}\right)^{3}} .
$$

Similarly, the derivative of each term, $p_{1}\left(z^{c}\right), p_{s}\left(z^{c}\right),\left(p_{1}\left(z^{c}\right)\right)^{\prime},\left(p_{s}\left(z^{c}\right)\right)^{\prime}$, with respect to each variable $z_{1}, \zeta, x_{s}$, is bounded by a comparable quantity, so there is some constant $c_{3}>0$ (depending on $m, n$ ) so that

$$
\begin{aligned}
& \max _{j=2, \ldots, m-1}\left\{\left|\frac{d p_{1}\left(z^{c}\right)}{d z_{1}}\right|_{\sigma}+\left|\frac{d\left(\left(p_{1}\left(z^{c}\right)\right)^{\prime}\right)}{d z_{1}}\right|_{\sigma}+\sum_{s=2}^{m-1}\left|\frac{d\left(\frac{1}{2}\left(p_{s}\left(z^{c}\right)+\left(p_{s}\left(z^{c}\right)\right)^{\prime}\right)\right)}{d z_{1}}\right|_{\sigma},\right. \\
&\left|\frac{d p_{1}\left(z^{c}\right)}{d \zeta}\right|_{\sigma}+\left|\frac{d\left(\left(p_{1}\left(z^{c}\right)\right)^{\prime}\right)}{d \zeta}\right|_{\sigma}+\sum_{s=2}^{m-1}\left|\frac{d\left(\frac{1}{2}\left(p_{s}\left(z^{c}\right)+\left(p_{s}\left(z^{c}\right)\right)^{\prime}\right)\right)}{d \zeta}\right|_{\sigma} \\
&\left.\left|\frac{d p_{1}\left(z^{c}\right)}{d x_{j}}\right|_{\sigma}+\left|\frac{d\left(\left(p_{1}\left(z^{c}\right)\right)^{\prime}\right)}{d x_{j}}\right|_{\sigma}+\sum_{s=2}^{m-1}\left|\frac{d\left(\frac{1}{2}\left(p_{s}\left(z^{c}\right)+\left(p_{s}\left(z^{c}\right)\right)^{\prime}\right)\right)}{d x_{j}}\right|_{\sigma}\right\} \\
& \leq c_{3}|\vec{e}|_{r} /\left(r-r^{\prime}\right)^{3} \leq c_{3} \delta_{3} \leq \frac{1}{2}
\end{aligned}
$$

if $\delta_{3} \leq 1 /\left(2 c_{3}\right)$. It also follows from Corollary 5.8 that 


$$
\begin{aligned}
\left|p_{1}\left(z^{c}\right)\right|_{r^{\prime}}+\left|\left(p_{1}\left(z^{c}\right)\right)^{\prime}\right|_{r^{\prime}}+\sum_{s=2}^{m-1}\left|\frac{1}{2}\left(p_{s}\left(z^{c}\right)+\left(p_{s}\left(z^{c}\right)\right)^{\prime}\right)\right|_{r^{\prime}} \\
\quad \leq 2\left\|p_{1}\right\|_{\rho}+\sum_{s=2}^{m-1}\left\|p_{s}\right\|_{\rho} \leq \frac{m c_{1}|\vec{e}|_{r}}{(r-\rho)^{2}} \leq \frac{m c_{1} \delta_{3}\left(r-r^{\prime}\right)^{3}}{(r-\rho)^{2}} \\
\quad=9 m c_{1} \delta_{3}\left(r-r^{\prime}\right) \leq \frac{1}{2}\left(\sigma-r^{\prime}\right)
\end{aligned}
$$

if $\delta_{3} \leq 1 /\left(54 m c_{1}\right)$. So, by Lemma 6.3 , given $\left(\tilde{z}_{1}, \tilde{\zeta}, \tilde{x}\right) \in D_{r^{\prime}}$, there exists a unique $\left(z_{1}, \zeta, x\right) \in D_{\sigma}$ such that $\left(\tilde{z}_{1}, \tilde{\zeta}, \tilde{x}\right)=\tau\left(z_{1}, \zeta, x\right)$.

By inspection of the form of $\tau$, if $\left(z_{1}, \zeta, x\right) \in D_{\sigma}$ and $\tau\left(z_{1}, \zeta, x\right)=\left(\tilde{z}_{1}, \tilde{\zeta}, \tilde{x}\right)$, then $\tau\left(\bar{\zeta}, \bar{z}_{1}, \bar{x}\right)=\left(\overline{\tilde{\zeta}}, \overline{\tilde{z}}_{1}, \overline{\tilde{x}}\right)$. If, further, $\left(\tilde{z}_{1}, \tilde{\zeta}, \tilde{x}\right)=\left(\overline{\tilde{\zeta}}, \overline{\tilde{z}}_{1}, \overline{\tilde{x}}\right) \in D_{r^{\prime}}$, then $\left(z_{1}, \zeta, x\right)=$ $\left(\bar{\zeta}, \bar{z}_{1}, \bar{x}\right)$ by uniqueness of the inverse. In particular, if $\left|\tilde{z}_{1}\right|<r^{\prime}$ and for $s=$ $2, \ldots, m-1, \tilde{x}_{s}$ is real and $\left|\tilde{x}_{s}\right|<r^{\prime}$, then $\phi\left(\tilde{z}_{1}, \overline{\tilde{z}}_{1}, \tilde{x}\right)$ is of the form $\left(z_{1}, \bar{z}_{1}, x\right)$ for some $z_{1}$ with $\left|z_{1}\right|<\sigma$ and $x$ real with $\left|x_{s}\right|<\sigma$. Such $\left(z_{1}, x\right)$ is unique, given $\left(\tilde{z}_{1}, \tilde{x}\right)$ : suppose there were $\left(z_{1}^{0}, x^{0}\right)$ with $\left|z_{1}^{0}\right|<\sigma,\left|x_{s}^{0}\right|<\sigma, x^{0}$ real, such that

$$
\begin{aligned}
& \tilde{z}_{1}=\tau_{1}\left(z_{1}^{0}, \overline{z_{1}^{0}}, x^{0}\right), \\
& \tilde{x}_{s}=\tau_{s+1}\left(z_{1}^{0}, \overline{z_{1}^{0}}, x^{0}\right) \quad \text { for } s=2, \ldots, m-1 .
\end{aligned}
$$

Then the second component $\tau_{2}\left(z_{1}^{0}, \overline{z_{1}^{0}}, x^{0}\right)$ can be calculated to have some value $\tilde{\zeta}$, so $\tau\left(z_{1}^{0}, \overline{z_{1}^{0}}, x^{0}\right)=\left(\tilde{z}_{1}, \tilde{\zeta}, \tilde{x}\right)$. By the formula for $\tau, \tilde{\zeta}=\overline{\tilde{z}}_{1}$, so $\left(\tilde{z}_{1}, \tilde{\zeta}, \tilde{x}\right) \in D_{r^{\prime}}$ and $\left(z_{1}^{0}, \bar{z}_{1}^{0}, x^{0}\right)=\phi\left(\tilde{z}_{1}, \tilde{\zeta}, \tilde{x}\right)=\phi\left(\tilde{z}_{1}, \overline{\tilde{z}}_{1}, \tilde{x}\right)=\left(z_{1}, \bar{z}_{1}, x\right)$, so we can conclude from the uniqueness of Lemma 6.3 that $z_{1}^{0}=z_{1}$ and $x^{0}=x$.

Theorem 6.6. There exist constants $c_{4}>0$ and $\delta_{4}>0$ (depending on $m, n$ ) such that for any $\frac{1}{2}<r^{\prime}<r \leq 1$ (with $\sigma, \rho$ as in the previous theorem), and any $\vec{e}$ as in Corollary 5.7 with $|\vec{e}|_{r} \leq \delta_{4}\left(r-r^{\prime}\right)^{3}$, there exist a holomorphic map

$$
\Psi: \Delta_{\rho} \rightarrow \mathbb{C}^{n}, \quad\left(z_{1}, \ldots, z_{n}\right) \mapsto\left(\tilde{z}_{1}, \ldots, \tilde{z}_{n}\right),
$$

with a holomorphic inverse $\psi: \Delta_{\sigma} \rightarrow \Delta_{\rho}$, and a holomorphic map $\tilde{e}=\left(\tilde{E}_{2}, \ldots, \tilde{e}_{n}\right)$ from $D_{r^{\prime}}$ to $\mathbb{C}^{n-1}$, such that the defining equations for $M$ are

$$
\begin{aligned}
\tilde{y}_{s} & =\tilde{E}_{s}\left(\tilde{z}_{1}, \overline{\tilde{z}}_{1}, \tilde{x}\right), \\
\tilde{z}_{t} & =\overline{\tilde{z}}_{1}\left(\tilde{x}_{2(t-m+2)}+i \tilde{x}_{2(t-m+2)+1}\right)+\tilde{e}_{t}\left(\tilde{z}_{1}, \overline{\tilde{z}}_{1}, \tilde{x}\right), \\
\tilde{z}_{n-1} & =\overline{\tilde{z}}_{1}^{2}+\tilde{e}_{n-1}\left(\tilde{z}_{1}, \overline{\tilde{z}}_{1}, \tilde{x}\right), \\
\tilde{z}_{n} & =\overline{\tilde{z}}_{1}\left(\tilde{z}_{1}+\tilde{x}_{2}+i \tilde{x}_{3}\right)+\tilde{e}_{n}\left(\tilde{z}_{1}, \overline{\tilde{z}}_{1}, \tilde{x}\right),
\end{aligned}
$$

for $\left|\tilde{z}_{1}\right|<r^{\prime},\left|\tilde{x}_{s}\right|<r^{\prime}$. The degree of $\tilde{e}$ is at least $2 d-2$, and

$$
|\tilde{e}|_{r^{\prime}} \leq \frac{c_{4}|\vec{e}|_{r}^{2}}{\left(r-r^{\prime}\right)^{3}}
$$


Proof. Initially, choose $\delta_{4} \leq \min \left\{\frac{8}{3} \delta_{1}, \frac{8}{27} \delta_{2}, \delta_{3}\right\}$, so that Theorems 6.2, 6.4, 6.5 apply, and define $\Psi, \psi, \vec{q}$, and $\phi$ in terms of the given $\vec{e}$ and the functions $\vec{p}$ constructed in Corollary 5.7. Define $\tilde{e}$ to be the composite of holomorphic maps $\vec{q} \circ \phi: D_{r^{\prime}} \rightarrow \mathbb{C}^{n-1}$, so that by Theorem 6.2,

$$
|\tilde{e}|_{r^{\prime}} \leq|\vec{q}|_{\sigma} \leq \frac{c_{2}|\vec{e}|_{r}^{2}}{(r-\sigma)^{3}}=\frac{c_{2}|\vec{e}|_{r}^{2}}{\left(\frac{2}{3}\left(r-r^{\prime}\right)\right)^{3}} .
$$

Since $\phi\left(\tilde{z}_{1}, \overline{\tilde{z}}_{1}, \tilde{x}\right)$ has no constant terms, and $\vec{q}$ has degree $\geq 2 d-2$ by construction, $\tilde{e}\left(\tilde{z}_{1}, \overline{\tilde{z}}_{1}, \tilde{x}\right)$ also has degree at least $2 d-2$.

Given $\tilde{z}_{1}, \tilde{x}$ such that $\left|\tilde{z}_{1}\right|<r^{\prime}$, and $\tilde{x}$ is real with $\left|\tilde{x}_{s}\right|<r^{\prime}$, define quantities $\tilde{z}_{2}, \ldots, \tilde{z}_{n}$ by

$$
\begin{aligned}
\tilde{z}_{s} & =\tilde{x}_{s}+i \tilde{E}_{s}\left(\tilde{z}_{1}, \overline{\tilde{z}}_{1}, \tilde{x}\right), \\
\tilde{z}_{t} & =\overline{\tilde{z}}_{1}\left(\tilde{x}_{2(t-m+2)}+i \tilde{x}_{2(t-m+2)+1}\right)+\tilde{e}_{t}\left(\tilde{z}_{1}, \overline{\tilde{z}}_{1}, \tilde{x}\right) \\
\tilde{z}_{n-1} & =\overline{\tilde{z}}_{1}^{2}+\tilde{e}_{n-1}\left(\tilde{z}_{1}, \overline{\tilde{z}}_{1}, \tilde{x}\right), \\
\tilde{z}_{n} & =\overline{\tilde{z}}_{1}\left(\tilde{z}_{1}+\tilde{x}_{2}+i \tilde{x}_{3}\right)+\tilde{e}_{n}\left(\tilde{z}_{1}, \overline{\tilde{z}}_{1}, \tilde{x}\right),
\end{aligned}
$$

and define $\tilde{z}=\left(\tilde{z}_{1}, \tilde{z}_{2}, \ldots, \tilde{z}_{n}\right)$. The claim of the theorem is that $\psi(\tilde{z}) \in M$.

If $\delta_{4}^{2} \leq 32 /\left(81 c_{2}\right)$, then

$$
|\tilde{e}|_{r^{\prime}} \leq \frac{c_{2}\left(\delta_{4}\left(r-r^{\prime}\right)^{3}\right)^{2}}{(r-\sigma)^{3}}=c_{2}\left(\delta_{4}\right)^{2} \frac{3^{6}}{2^{3}}\left(\sigma-r^{\prime}\right)^{3} \leq c_{2}\left(\delta_{4}\right)^{2} \frac{3^{6}}{2^{3} 6^{2}}\left(\sigma-r^{\prime}\right) \leq \sigma-r^{\prime},
$$

so $\tilde{z} \in \Delta_{\sigma}$, the domain of $\psi$.

By Theorem 6.5, there exists a unique $\left(z_{1}, x\right)$ (the first and last components of $\left.\left(z_{1}, \bar{z}_{1}, x\right)=\phi\left(\tilde{z}_{1}, \overline{\tilde{z}}_{1}, \tilde{x}\right)\right)$ such that $\left|z_{1}\right|<\sigma, x$ is real with $\left|x_{s}\right|<\sigma$, and

$$
\begin{aligned}
& \tilde{z}_{1}=z_{1}+p_{1}\left(z_{1}, x_{2}+i E_{2}\left(z_{1}, \bar{z}_{1}, x\right), \ldots, \bar{z}_{1}\left(z_{1}+x_{2}+i x_{3}\right)+e_{n}\left(z_{1}, \bar{z}_{1}, x\right)\right), \\
& \tilde{x}_{s}=x_{s}+\operatorname{Re}\left(p_{s}\left(z_{1}, x_{2}+i E_{2}\left(z_{1}, \bar{z}_{1}, x\right), \ldots, \bar{z}_{1}\left(z_{1}+x_{2}+i x_{3}\right)+e_{n}\left(z_{1}, \bar{z}_{1}, x\right)\right)\right) .
\end{aligned}
$$

Then define quantities $z_{2}, \ldots, z_{n}$ by

$$
\begin{aligned}
z_{s} & =x_{s}+i E_{s}\left(z_{1}, \bar{z}_{1}, x\right), \\
z_{t} & =\bar{z}_{1}\left(x_{2(t-m+2)}+i x_{2(t-m+2)+1}\right)+e_{t}\left(z_{1}, \bar{z}_{1}, x\right), \\
z_{n-1} & =\bar{z}_{1}^{2}+e_{n-1}\left(z_{1}, \bar{z}_{1}, x\right), \\
z_{n} & =\bar{z}_{1}\left(z_{1}+x_{2}+i x_{3}\right)+e_{n}\left(z_{1}, \bar{z}_{1}, x\right),
\end{aligned}
$$

and define, as in (11) and (24), $\vec{z}=\left(z_{1}, x, \ldots, \bar{z}_{1}\left(z_{1}+x_{2}+i x_{3}\right)\right)$ and $\vec{z}+\vec{e}=$ $\left(z_{1}, z_{2}, \ldots, z_{n}\right)$ as in (8) and (25). Since $\left|z_{1}\right|<\sigma<r$ and $\left|x_{s}\right|<\sigma<r, \vec{z}+\vec{e} \in M$, 
and if $\delta_{4} \leq \frac{4}{3}$, then

$$
|\vec{e}|_{\sigma} \leq|\vec{e}|_{r} \leq \delta_{4}\left(r-r^{\prime}\right)^{3}=\delta_{4} \cdot 27(\rho-\sigma)^{3}<\delta_{4} \frac{27}{6^{2}}(\rho-\sigma) \leq(\rho-\sigma),
$$

so $\vec{z}+\vec{e} \in \Delta_{\rho}$, which is contained in the domain of $\vec{p}$.

Next, by the construction of $\vec{q}, \tilde{e}$, and $\tilde{z}$, we see that $\Psi(\vec{z}+\vec{e})$ equals

$$
\begin{aligned}
& \left(z_{1}+p_{1}(\vec{z}+\vec{e}), \ldots, z_{n}+p_{n}(\vec{z}+\vec{e})\right) \\
& =\left(\tilde{z}_{1}, \ldots, \tilde{x}_{s}+i E_{s}\left(z_{1}, \bar{z}_{1}, x\right)+i \operatorname{Im} p_{s}(\vec{z}+\vec{e}), \ldots,\right. \\
& \overline{\tilde{z}_{1}-p_{1}(\vec{z}+\vec{e})}\left(\tilde{x}_{2(t-m+2)}-\operatorname{Re} p_{2(t-m+2)}(\vec{z}+\vec{e})\right) \\
& +i \overline{\tilde{z}_{1}-p_{1}(\vec{z}+\vec{e})}\left(\tilde{x}_{2(t-m+2)+1}-\operatorname{Re} p_{2(t-m+2)+1}(\vec{z}+\vec{e})\right) \\
& +e_{t}\left(z_{1}, \bar{z}_{1}, x\right)+p_{t}(\vec{z}+\vec{e}), \ldots,
\end{aligned}
$$

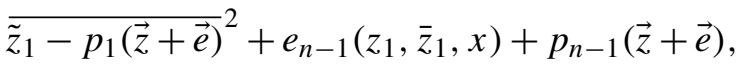

$$
\begin{aligned}
& \overline{\tilde{z}_{1}-p_{1}(\vec{z}+\vec{e})}\left(\tilde{z}_{1}-p_{1}(\vec{z}+\vec{e})+\tilde{x}_{2}-\operatorname{Re} p_{2}(\vec{z}+\vec{e})+i \tilde{x}_{3}-i \operatorname{Re} p_{3}(\vec{z}+\vec{e})\right) \\
& =\left(\tilde{z}_{1}, \ldots, \tilde{x}_{s}+i Q_{s}\left(z_{1}, \bar{z}_{1}, x\right), \ldots,\right. \\
& \left.+e_{n}\left(z_{1}, \bar{z}_{1}, x\right)+p_{n}(\vec{z}+\vec{e})\right) \\
& \overline{\tilde{z}}_{1}\left(\tilde{x}_{2(t-m+2)}+i \tilde{x}_{2(t-m+2)+1}\right)+q_{t}\left(z_{1}, \bar{z}_{1}, x\right), \ldots, \\
& \left.\overline{\tilde{z}}_{1}^{2}+q_{n-1}\left(z_{1}, \bar{z}_{1}, x\right), \overline{\tilde{z}}_{1}\left(\tilde{z}_{1}+\tilde{x}_{2}+i \tilde{x}_{3}\right)+q_{n}\left(z_{1}, \bar{z}_{1}, x\right)\right) \\
& =\left(\tilde{z}_{1}, \ldots, \tilde{x}_{s}+i Q_{s}\left(\phi\left(\tilde{z}_{1}, \overline{\tilde{z}}_{1}, \tilde{x}\right)\right), \ldots,\right. \\
& \overline{\tilde{z}}_{1}\left(\tilde{x}_{2(t-m+2)}+i \tilde{x}_{2(t-m+2)+1}\right)+q_{t}\left(\phi\left(\tilde{z}_{1}, \overline{\tilde{z}}_{1}, \tilde{x}\right)\right), \ldots, \\
& \left.\overline{\tilde{z}}_{1}^{2}+q_{n-1}\left(\phi\left(\tilde{z}_{1}, \overline{\tilde{z}}_{1}, \tilde{x}\right)\right), \overline{\tilde{z}}_{1}\left(\tilde{z}_{1}+\tilde{x}_{2}+i \tilde{x}_{3}\right)+q_{n}\left(\phi\left(\tilde{z}_{1}, \overline{\tilde{z}}_{1}, \tilde{x}\right)\right)\right) \\
& =\left(\tilde{z}_{1}, \ldots, \tilde{x}_{s}+i \tilde{E}_{s}\left(\tilde{z}_{1}, \overline{\tilde{z}}_{1}, \tilde{x}\right), \ldots\right. \text {, } \\
& \overline{\tilde{z}}_{1}\left(\tilde{x}_{2(t-m+2)}+i \tilde{x}_{2(t-m+2)+1}\right)+\tilde{e}_{t}\left(\tilde{z}_{1}, \overline{\tilde{z}}_{1}, \tilde{x}\right), \ldots, \\
& \left.\overline{\tilde{z}}_{1}^{2}+\tilde{e}_{n-1}\left(\tilde{z}_{1}, \overline{\tilde{z}}_{1}, \tilde{x}\right), \overline{\tilde{z}}_{1}\left(\tilde{z}_{1}+\tilde{x}_{2}+i \tilde{x}_{3}\right)+\tilde{e}_{n}\left(\tilde{z}_{1}, \overline{\tilde{z}}_{1}, \tilde{x}\right)\right) \\
& =\tilde{z} \text {. }
\end{aligned}
$$

Here we have used the fact that $\vec{p}$ is a solution of (10)-(11). By the uniqueness of Theorem 6.4, $\psi(\tilde{z})=\vec{z}+\vec{e}$ lies in $M$.

\section{Composition of approximate solutions}

The previous theorem's quadratic estimate on the size of $\tilde{e}$ in terms of $\vec{e}$ allows for the rapid convergence of a sequence of approximations. A couple of technical lemmas will be needed to measure the behavior of composite mappings. Theorem 7.7, which is the last step in proving Proposition 3.3, uses these lemmas and the 
estimates of the previous section to prove convergence of a sequence of transformations, following the ideas of [Moser 1985].

Notation 7.1. For $R_{1}>0$ and a $n \times n$ matrix of complex-valued functions $F=$ $\left(F_{k j}(\vec{z})\right)$ on $\Delta_{R_{1}}$, define

$$
\|F\|_{R_{1}}=\max _{j=1, \ldots, n}\left\{\sum_{k=1}^{n} \sup _{\vec{z} \in \Delta_{R_{1}}}\left|F_{k j}(\vec{z})\right|\right\} .
$$

This "maximum column sum" norm has already appeared, in Corollary 5.8 and Lemmas 6.1 and 6.3, in the case where $F=\mathrm{D} f=\mathrm{D}_{\vec{z}} f$, the Jacobian matrix of some map $f: \Delta_{R_{1}} \rightarrow \mathbb{C}^{n}$ at $\vec{z} \in \Delta_{R_{1}}$. The $3 \times 3$ case of the following lemma was proved in [Coffman 2004].

Lemma 7.2. If $\|A\|_{R_{1}}<1$, then $\mathbb{1}+A$ is invertible (where $\mathbb{1}$ is the $n \times n$ identity matrix), and

$$
\left\|(\mathbb{1}+A)^{-1}\right\|_{R_{1}} \leq \frac{1}{1-\|A\|_{R_{1}}} .
$$

We need an elementary fact from the calculus of one real variable: Lemma 7.3. If $\mu_{k}$ is a sequence such that $0 \leq \mu_{k}<1$ and $\sum_{k=0}^{\infty} \mu_{k}$ is a convergent
series, then the sequence of partial products

$$
\prod_{k=0}^{N} \frac{1}{1-\mu_{k}}
$$

is bounded above by some positive limit.

Notation 7.4. For $v=0,1,2, \ldots$, define a sequence $\left\{1, \frac{3}{4}, \frac{4}{6}, \frac{5}{8}, \ldots\right\}$ by the formula

$$
r_{\nu}=\frac{1}{2}\left(1+\frac{1}{v+1}\right) .
$$

Note that $\frac{1}{2}<r_{v} \leq 1$, and the sequence is decreasing, with

$$
r_{v}-r_{v+1}=\frac{1}{2(v+1)(v+2)} \quad \text { and } \quad \frac{r_{v+1}-r_{v+2}}{r_{v}-r_{v+1}}=\frac{v+1}{v+3} \geq \frac{1}{3} .
$$

Notation 7.5. Define $\sigma_{v}=r_{v+1}+\frac{1}{3}\left(r_{v}-r_{v+1}\right), \rho_{v}=r_{v+1}+\frac{2}{3}\left(r_{v}-r_{v+1}\right)$, as in Theorem 6.5.

Recall that given $\eta>0$, there is some scaling transformation so that $M \cap \Delta_{1}$ is defined by (5), with $\vec{e}$ holomorphic on $D_{1}$, degree $d \geq 3$, and $|\vec{e}|_{1} \leq \eta$.

Notation 7.6. Set $\vec{e}_{0}=\vec{e}$ (so $\left|\vec{e}_{0}\right|_{r_{0}}=|\vec{e}|_{1} \leq \eta$ ), and inductively define the formal series $\vec{e}_{v+1}\left(z_{1}, \zeta, x\right)$ in terms of $\vec{e}_{v}\left(z_{1}, \zeta, x\right)$, by the $\vec{e} \mapsto \tilde{e}$ procedure of Theorem 6.6, 
with $r=r_{\nu}, r^{\prime}=r_{v+1}$. Each $\vec{e}_{\nu}$ defines, as in the previous theorems, functions $\vec{p}_{\nu}$, $\vec{q}_{\nu}, \Psi_{\nu}, \psi_{\nu}, \phi_{\nu}$, and the degree of $\vec{e}_{v}$ is denoted $d_{\nu}$.

Also recall that the degree $d_{v+1}$ of $\vec{e}_{v+1}$ is at least $2 d_{v}-2$; it can be checked that this, together with $d_{0}=d \geq 3$, implies $d_{v} \geq 2^{v}+2$.

The plan is to show that the bound for $\vec{e}_{v}$ in the hypothesis of Theorem 6.6 holds for all $v$, to get a sequence of transformations $\psi_{v}: \Delta_{\sigma_{v}} \rightarrow \Delta_{\rho_{v}}$, so that the composition

$$
\psi_{0} \circ \ldots \circ \psi_{\nu-1} \circ \psi_{\nu}: \Delta_{\sigma_{v}} \rightarrow \Delta_{\rho_{0}}
$$

is well-defined, $\vec{e}_{v}$ is holomorphic on $D_{r_{v}}$, and $\lim _{\nu \rightarrow \infty}\left|\vec{e}_{\nu}\right|_{r_{v}}=0$.

Theorem 7.7. There exists $\eta>0$ (depending on $m, n$ ) so that if $\vec{e}_{0}$ and $M$ are as described above, then there exists a holomorphic transformation $\psi: \Delta_{1 / 2} \rightarrow \mathbb{C}^{n}$, with a holomorphic inverse $\Psi$, and such that if $\tilde{z} \in \tilde{M} \cap \Delta_{1 / 2}$, then $\psi(\tilde{z}) \in M$.

Proof. Set $\delta_{5}=\min \left\{\delta_{4}, 1 /\left(27 c_{4}\right)\right\}$ and choose $0<\eta<\min \left\{\delta_{5} / 64,1 /\left(1728 c_{1}\right)\right\}$. It will be shown that $\left|\vec{e}_{v}\right|_{r_{v}} \leq \delta_{5}\left(r_{v}-r_{v+1}\right)^{3}$ implies $\left|\vec{e}_{v+1}\right|_{r_{v+1}} \leq \delta_{5}\left(r_{v+1}-r_{v+2}\right)^{3}$. By Theorem 6.6, $\left|\vec{e}_{v}\right|_{r_{v}} \leq \delta_{4}\left(r_{v}-r_{v+1}\right)^{3}$ and $\left|\vec{e}_{v}\right|_{r_{v}} \leq\left(r_{v}-r_{v+1}\right)^{3} /\left(27 c_{4}\right)$ imply

$$
\left|\vec{e}_{v+1}\right|_{r_{v+1}} \leq \frac{c_{4}\left|\vec{e}_{v}\right|_{r_{v}}^{2}}{\left(r_{v}-r_{v+1}\right)^{3}} \leq \frac{1}{27}\left|\vec{e}_{v}\right|_{r_{v}}
$$

this already suggests a geometric decrease in the sequence of norms. Then, using the properties of the sequence $r_{v}$,

$$
\frac{1}{27}\left|\vec{e}_{v}\right|_{r_{v}} \leq \frac{1}{27} \delta_{5}\left(r_{v}-r_{v+1}\right)^{3} \leq \delta_{5}\left(r_{v+1}-r_{v+2}\right)^{3},
$$

which proves the claimed implication. Using this as an inductive step, and starting the induction with $\left|\vec{e}_{0}\right|_{r_{0}} \leq \eta<\frac{1}{64} \delta_{5}=\delta_{5}\left(r_{0}-r_{1}\right)^{3}$, the hypothesis of Theorem 6.6 is satisfied for all $v$. The first of three conclusions from Theorem 6.6 is that $\vec{e}_{v}$ is holomorphic on $D_{r_{v}}$, with degree $d_{v} \geq 2^{v}+2$, and $\left|\vec{e}_{v}\right|_{r_{v}} \leq 27^{-v} \eta$. Secondly, $\psi_{0} \circ \ldots \circ \psi_{\nu}$ is a well-defined holomorphic map $\Delta_{\sigma_{v}} \rightarrow \Delta_{\rho_{0}}$, and $\Psi_{v} \circ \ldots \circ \Psi_{0}$ is well-defined and holomorphic on the image $\left(\psi_{0} \circ \ldots \circ \psi_{\nu}\right)\left(\Delta_{\sigma_{v}}\right)$, so that

$$
\Psi_{v} \circ \ldots \circ \Psi_{0} \circ \psi_{0} \circ \ldots \circ \psi_{v}
$$

is the identity on $\Delta_{\sigma_{v}}$. The third conclusion is that if $\left|\tilde{z}_{1}\right|<r_{v+1}$ and $\left|\tilde{x}_{s}\right|<r_{\nu+1}$, and $\tilde{z}$ is defined as in (27) with $\tilde{e}=\vec{e}_{\nu+1}$, then $\left(\psi_{0} \circ \ldots \circ \psi_{\nu}\right)(\tilde{z}) \in M$. For any $\vec{z}=\left(z_{1}, \ldots, z_{n}\right) \in \Delta_{1 / 2}$, the sequence (depending on $\left.v\right)\left(\psi_{0} \circ \ldots \circ \psi_{v-1} \circ \psi_{v}\right)(\vec{z})$ is contained in $\Delta_{\rho_{0}}=\Delta_{11 / 12}$. The following argument, beginning with several applications of Lemma 6.1, shows this sequence is a Cauchy sequence, and converges to some value $\psi(\vec{z})$. 
We have

$$
\begin{aligned}
\sum_{k=1}^{n}\left|\left(\psi_{0} \circ \ldots \circ \psi_{\nu+1}\right)_{k}(\vec{z})-\left(\psi_{0} \circ \ldots \circ \psi_{\nu}\right)_{k}(\vec{z})\right| \\
\quad=\sum_{k=1}^{n}\left|\left(\psi_{0}\right)_{k}\left(\left(\psi_{1} \circ \ldots \circ \psi_{v+1}\right)(\vec{z})\right)-\left(\psi_{0}\right)_{k}\left(\left(\psi_{1} \circ \ldots \circ \psi_{v}\right)(\vec{z})\right)\right| \\
\leq\|\| \mathrm{D} \psi_{0} \|_{\rho_{1}} \cdot \sum_{j=1}^{n}\left|\left(\psi_{1} \circ \ldots \circ \psi_{\nu+1}\right)_{j}(\vec{z})-\left(\psi_{1} \circ \ldots \circ \psi_{v}\right)_{j}(\vec{z})\right| \\
\leq\left(\prod_{\ell=0}^{v} \mid\left\|\mathrm{D} \psi_{\ell}\right\|_{\rho_{\ell+1}}\right) \cdot \sum_{j=1}^{n}\left|\left(\psi_{\nu+1}\right)_{j}(\vec{z})-z_{j}\right|
\end{aligned}
$$

By the estimate from Lemma 6.3, with $f=\vec{p}_{v+1}$ and $K=\frac{1}{2}$ from the proof of Theorem 6.4, and then using the bound for $\vec{p}$ from Corollary 5.8,

$$
\begin{aligned}
\sum_{j=1}^{n}\left|\left(\psi_{v+1}\right)_{j}(\vec{z})-z_{j}\right| & \leq \frac{1}{1-\frac{1}{2}} \sum_{j=1}^{n}\left|\left(\vec{p}_{v+1}\right)_{j}(\vec{z})\right| \leq 2 \sum_{j=1}^{n}\left\|\left(\vec{p}_{v+1}\right)_{j}\right\|_{1 / 2} \\
& \leq 2 \sum_{j=1}^{n}\left\|\left(\vec{p}_{v+1}\right)_{j}\right\|_{\rho_{v+1}} \leq 2 n \frac{c_{1}\left|\vec{e}_{v+1}\right|_{r_{v+1}}}{\left(r_{v+1}-\rho_{v+1}\right)^{2}} \\
& =18 n \frac{c_{1}\left|\vec{e}_{v+1}\right|_{r_{v+1}}}{\left(r_{v+1}-r_{v+2}\right)^{2}}=72 n c_{1}(v+2)^{2}(v+3)^{2}\left|\vec{e}_{v+1}\right|_{r_{v+1}} \\
& \leq \frac{72 n c_{1}(v+2)^{2}(v+3)^{2} \eta}{27^{v+1}}
\end{aligned}
$$

It follows from $\mathrm{D}_{\vec{z}} \psi_{\ell}=\left(\mathbb{1}+\mathrm{D}_{\psi_{\ell}(\vec{z})} \vec{p}_{\ell}\right)^{-1}$ and Lemma 7.2 that

$$
\left\|\left|\mathrm{D} \psi_{\ell}\left\|_{\rho_{\ell+1}}=\right\|\right|\left(\mathbb{1}+\mathrm{D}_{\psi_{\ell}(\vec{z})} \vec{p}_{\ell}\right)^{-1} \mid\right\|_{\rho_{\ell+1}} \leq\left\|\left(\mathbb{1}+\mathrm{D} \vec{p}_{\ell}\right)^{-1}\right\|_{\rho_{\ell}} \leq \frac{1}{1-\left\|\mathrm{D} \vec{p}_{\ell}\right\|_{\rho_{\ell}}} .
$$

Then, by Lemma 7.3, the product from (28) is bounded above by some constant $c_{5}>0$, since by Corollary 5.8,

$$
\begin{aligned}
\sum_{\ell=0}^{\infty}\|\| \mathrm{D} \vec{p}_{\ell} \mid \|_{\rho_{\ell}} & \leq \sum_{\ell=0}^{\infty} \frac{c_{1}\left|\vec{e}_{\ell}\right|_{r_{\ell}}}{\left(r_{\ell}-\rho_{\ell}\right)^{3}}=\sum_{\ell=0}^{\infty} \frac{27 c_{1}\left|\vec{e}_{\ell}\right|_{r_{\ell}}}{\left(r_{\ell}-r_{\ell+1}\right)^{3}} \\
& =\sum_{\ell=0}^{\infty} 216(\ell+1)^{3}(\ell+2)^{3} c_{1}\left|\vec{e}_{\ell}\right|_{r_{\ell}} \leq \sum_{\ell=0}^{\infty} \frac{216(\ell+1)^{3}(\ell+2)^{3} c_{1} \eta}{27^{\ell}}
\end{aligned}
$$

a convergent infinite series with terms less than 1 . 
The inequality

$$
\sum_{k=1}^{n}\left|\left(\psi_{0} \circ \ldots \circ \psi_{v+1}\right)_{k}(\vec{z})-\left(\psi_{0} \circ \ldots \circ \psi_{v}\right)_{k}(\vec{z})\right| \leq \frac{72 n c_{1} c_{5}(v+2)^{2}(v+3)^{2} \eta}{27^{v+1}}
$$

is enough to show that the sequence of composite functions converges pointwise and uniformly to a function $\psi$ on $\Delta_{1 / 2}$.

Remark. Although some details remain to be checked, it seems plausible that a similar rapid convergence argument could be used to prove an analogous analytic stability property for a nondegenerate CR singularity of a real 3-manifold in $\mathbb{C}^{4}$, as conjectured in [Coffman 2006].

\section{Analogy with singularity theory}

To continue with the theme of analogies between the normal form result and the properties of Whitney's cross-cap singularity, we briefly consider the notion of complexification. If the defining equations of a real $m$-submanifold $M$ in $\mathbb{C}^{n}$ with a CR singularity at $\overrightarrow{0}$ are given as a graph over the tangent space as in (1), then $M$ can also be considered as the image of a real analytic parametrization

$$
\pi: \mathbb{R}^{m} \rightarrow \mathbb{R}^{2 n}, \quad(z, x) \mapsto\left(z, x, H_{s}(z, x), h_{u}(z, x)\right) .
$$

Then the spaces $\mathbb{R}^{m}, \mathbb{R}^{2 n}$ can be embedded as totally real subspaces of $\mathbb{C}^{m}, \mathbb{C}^{2 n}$, and there is a complex analytic map $\pi_{c}: \mathbb{C}^{m} \rightarrow \mathbb{C}^{2 n}$ which restricts to $\pi$ on the totally real subspaces. In the following examples, composing with a projection $P: \mathbb{C}^{2 n} \rightarrow \mathbb{C}^{n}$ gives a holomorphic map $P \circ \pi_{c}$ which restricts to $\pi$ on the totally real $\mathbb{R}^{m}$ subspace, and its image is a complex subvariety of $\mathbb{C}^{n}$ containing $M$. Even though $\pi_{c}$ is an embedding, the composite $P \circ \pi_{c}$ can be singular, and the image of its critical point set contains the CR singular locus of $M$. For details and more examples of this construction, see [Webster 1985; Coffman 2002; Coffman 2003], and to be more precise, these maps should be considered only in some neighborhood of the origin in the domain and target.

Example 8.1. In the case $m=n=2$ [Bishop 1965], the local defining equation of a real surface with a nondegenerate $\mathrm{CR}$ singularity in $\mathbb{C}^{2}$ can be normalized to $z_{2}=\beta\left(z_{1}^{2}+\bar{z}_{1}^{2}\right)+z_{1} \bar{z}_{1}+O(3)$, where the coefficient $\beta \geq 0$ is a biholomorphic invariant. Considering the real embedding's quadratic part,

$$
\pi:\left(z_{1}, \bar{z}_{1}\right) \mapsto\left(z_{1}, \bar{z}_{1}, z_{2}=\beta\left(z_{1}^{2}+\bar{z}_{1}^{2}\right)+z_{1} \bar{z}_{1}, \bar{z}_{2}=\overline{\beta\left(z_{1}^{2}+\bar{z}_{1}^{2}\right)+z_{1} \bar{z}_{1}}\right)
$$

is a real analytic map from the totally real subspace $\left\{\left(z_{1}, w_{1}\right): w_{1}=\bar{z}_{1}\right\}$ of $\mathbb{C}^{2}$ to the totally real subspace $\left\{\left(z_{1}, w_{1}, z_{2}, w_{2}\right): w_{1}=\bar{z}_{1}, w_{2}=\bar{z}_{2}\right\}$ of $\mathbb{C}^{4}$, which extends 
to a complex analytic embedding

$$
\pi_{c}:\left(z_{1}, w_{1}\right) \mapsto\left(z_{1}, w_{1}, \beta\left(z_{1}^{2}+w_{1}^{2}\right)+z_{1} w_{1}, \beta\left(w_{1}^{2}+z_{1}^{2}\right)+w_{1} z_{1}\right) .
$$

Then composing with the projection $P: \mathbb{C}^{4} \rightarrow \mathbb{C}^{2}$ that forgets the $w_{1}, w_{2}$ variables in the target gives a map $P \circ \pi_{c}:(z, w) \mapsto\left(z, \beta\left(z^{2}+w^{2}\right)+z w\right)$. For $\beta>0$, this is a ramified two-to-one map onto $\mathbb{C}^{2}$ [Moser and Webster 1983; Webster 1985], and is analogous to Whitney's fold singularity $(x, y) \mapsto\left(x, y^{2}\right)$.

Example 8.2. An example of a cubic normal form for a $\mathrm{CR}$ singular surface in $\mathbb{C}^{2}$ in the $\beta=0$ case is $z_{2}=z_{1} \bar{z}_{1}+\bar{z}_{1}^{3}$ [Moser 1985]. The map $P \circ \pi_{c}:\left(z_{1}, w_{1}\right) \mapsto$ $\left(z_{1}, z_{1} w_{1}+w_{1}^{3}\right)$ is analogous to Whitney's cusp, $(x, y) \mapsto\left(x, x y+y^{3}\right)$.

Example 8.3. An example of a surface $M$ in $\mathbb{C}^{3}$ with a topologically unstable $\mathrm{CR}$ singularity, considered in [Coffman 2004], has real equations $z_{2}=\bar{z}_{1}^{2}, z_{3}=z_{1} \bar{z}_{1}$, which complexify to $P \circ \pi_{c}:\left(z_{1}, w_{1}\right) \mapsto\left(z_{1}, w_{1}^{2}, z_{1} w_{1}\right)$, exactly Whitney's normal form for the parametrization of the cross-cap singularity. The image of $P \circ \pi_{c}$ in $\mathbb{C}^{3}$ is $\left\{z_{1}^{2} z_{2}-z_{3}^{2}=0\right\}$, a singular complex hypersurface (Whitney's "umbrella" surface), and the smallest complex variety containing $M$.

Example 8.4. For the normal form variety $\widetilde{M}^{4,5}$, a parametrization $\mathbb{C}^{4} \rightarrow \mathbb{C}^{10} \rightarrow \mathbb{C}^{5}$ of the complexification is

$$
\left(z_{1}, w_{1}, z_{2}, z_{3}\right) \mapsto\left(z_{1}, z_{2}, z_{3}, w_{1}^{2}, w_{1}\left(z_{1}+z_{2}+i z_{3}\right)\right) .
$$

The real manifold $\widetilde{M}^{4,5}$ is the image of the restriction of this map to the totally real subspace $\left\{w_{1}=\bar{z}_{1}, z_{2}=\bar{z}_{2}, z_{3}=\bar{z}_{3}\right\}$ in the domain. The holomorphic map $\mathbb{C}^{4} \rightarrow \mathbb{C}^{5}$ parametrizes a singular complex hypersurface $\mathscr{H}$, which is the product of Whitney's cross-cap surface and a complex 2-plane, and the image $\left\{\left(z_{1}+z_{2}+i z_{3}\right)^{2} z_{4}-z_{5}^{2}=\right.$ $0\}$ is the smallest complex variety in $\mathbb{C}^{5}$ containing $\widetilde{M}^{4,5}$; a similar expression appeared in (15). The geometry of $\widetilde{M}^{4,5} \subseteq \mathscr{H}$ is considered in [Coffman 2003, §8], but with a different expression for the quadratic normal form.

Example 8.5. In general, the real variety $\widetilde{M}^{m, n}$ is contained in a singular subvariety of complex dimension $m$ in $\mathbb{C}^{n}$, the defining ideal of which contains, for example, $\left(z_{1}+z_{2}+i z_{3}\right)^{2} z_{n-1}-z_{n}^{2}$. As a consequence of Proposition 3.3, any real analytic $M$ is not a local uniqueness set for holomorphic functions in a neighborhood of a nondegenerate CR singularity; compare [Harris 1983].

For surfaces in $\mathbb{C}^{2}$, the two-to-one nature of the complexification $\mathbb{C}^{2} \rightarrow \mathbb{C}^{2}$ as in Example 8.1 was used in [Moser and Webster 1983] to solve a normal form problem in the $0<\beta<\frac{1}{2}$ case. Their methods are different from that of this paper; for example in the $(m, n)=(4,5)$ case, the map $\mathbb{C}^{4} \rightarrow \mathbb{C}^{5}$ from Example 8.4 is generally one-to-one, the two-to-one locus being contained in a complex subvariety in the domain as shown in [Coffman 2003]. 
Normal forms for the complexifications that look more like Whitney's monomial normal forms would be possible using a larger group, where the $z$ and $w$ variables could be transformed independently. Under the subgroup used to normalize the CR singularity, one expects equivalence classes of maps to be smaller, and continuous parameters ("moduli") to appear sooner (for more and for lower-order terms). However, invariants which distinguish maps under the larger group will still distinguish them under the smaller group. One may speculate that invariants of the complexification, such as the intrinsic derivative, the Boardman sequence, Jacobian extensions, etc., could provide a coarse but general beginning to the development of a CR singularity theory analogous to the singularity theory of maps [Golubitsky and Guillemin 1973; Porteous 1971].

\section{Acknowledgments}

The author thanks the referee(s) for comments on this, and also on an earlier version, which circulated as a preprint entitled Formal stability of the CR cross-cap, and which was based on [Coffman 1997]. The question of convergence answered by Proposition 3.3 was posed to the author by S. Webster.

\section{References}

[Ahlfors 1979] L. V. Ahlfors, Complex analysis, 3rd ed., McGraw-Hill, New York, 1979. MR 80c:30001 Zbl 0395.30001

[Baouendi et al. 2000] M. S. Baouendi, P. Ebenfelt, and L. Preiss Rothschild, "Local geometric properties of real submanifolds in complex space", Bull. Amer. Math. Soc. (N.S.) 37:3 (2000), 309336. MR 2001a:32043 Zbl 0955.32027

[Beloshapka 1997] V. K. Beloshapka, "The normal form of the germ of a four-dimensional real manifold in $\mathbb{C}^{5}$ at an $\mathbb{R C}$-singular point in general position", Mat. Zametki 61:6 (1997), 931-934. In Russian; translated in Math. Notes 61 (1997), 777-779. MR 99f:32012 Zbl 0917.32015

[Bishop 1965] E. Bishop, "Differentiable manifolds in complex Euclidean space”, Duke Math. J. 32 (1965), 1-21. MR 34 \#369 Zbl 0154.08501

[Coffman 1997] A. Coffman, Enumeration and normal forms of singularities in Cauchy-Riemann structures, dissertation, University of Chicago, 1997.

[Coffman 2002] A. Coffman, "CR singular immersions of complex projective spaces", Beiträge Algebra Geom. 43:2 (2002), 451-477. MR 2003k:32052 Zbl 1029.32020

[Coffman 2003] A. Coffman, "Real congruence of complex matrix pencils and complex projections of real Veronese varieties", Linear Algebra Appl. 370 (2003), 41-83. MR 2004f:14026 Zbl 1049.14042

[Coffman 2004] A. Coffman, "Analytic normal form for CR singular surfaces in $\mathbb{C}^{3}$ ", Houston J. Math. 30:4 (2004), 969-996. MR 2006d:32048 Zbl 1074.32013

[Coffman 2006] A. Coffman, "CR singularities of real threefolds in $\mathbb{C}^{4}$ ", Adv. Geom. 6:1 (2006), $109-137$.

[Domrin 1995a] A. V. Domrin, "On the number of RC-singular points of a four-dimensional real submanifold in a five-dimensional complex manifold", Mat. Zametki 57:2 (1995), 240-245, 318. In Russian; translated in Math. Notes 57 (1995), 167-170. MR 96d:32017 Zbl 0853.57028 
[Domrin 1995b] A. V. Domrin, "A description of characteristic classes of real submanifolds in complex manifolds in terms of RC-singularities", Izv. Ross. Akad. Nauk Ser. Mat. 59:5 (1995), 19-40. In Russian; translated in Math. Notes 59 (1995), 899-918. MR 97c:32020 Zbl 0877.57011

[Garrity 2000] T. Garrity, "Global structures on CR manifolds via Nash blow-ups", Michigan Math. J. 48 (2000), 281-294. MR 2001h:32058 Zbl 0995.32023

[Golubitsky and Guillemin 1973] M. Golubitsky and V. Guillemin, Stable mappings and their singularities, Graduate Texts in Math. 14, Springer, New York, 1973. MR 49 \#6269 Zbl 0294.58004 [Haefliger 1961] A. Haefliger, "Plongements différentiables de variétés dans variétés", Comment. Math. Helv. 36 (1961), 47-82. MR 26 \#3069 Zbl 0102.38603

[Harris 1981] G. A. Harris, “Geometry near a C.R. singularity”, Illinois J. Math. 25:1 (1981), 147158. MR 83g:32020 Zbl 0438.32003

[Harris 1983] G. A. Harris, "Real-analytic submanifolds which are local uniqueness sets for holomorphic functions of $\mathbb{C}^{3}$ ", Trans. Amer. Math. Soc. 277:1 (1983), 343-351. MR 84f:32005 Zbl 0512.32014

[Moser 1985] J. Moser, "Analytic surfaces in $\mathbb{C}^{2}$ and their local hull of holomorphy", Ann. Acad. Sci. Fenn. Ser. A I Math. 10 (1985), 397-410. MR 87c:32024 Zbl 0585.32007

[Moser and Webster 1983] J. K. Moser and S. M. Webster, "Normal forms for real surfaces in $\mathbb{C}^{2}$ near complex tangents and hyperbolic surface transformations", Acta Math. 150:3-4 (1983), 255296. MR 85c:32034 Zbl 0519.32015

[Porteous 1971] I. R. Porteous, "Simple singularities of maps", pp. 286-307 in Proceedings of Singularities Symposium (Liverpool, 1969/70), vol. I, edited by C. T. C. Wall, Lecture Notes in Math. 192, Springer, Berlin, 1971. MR 45 \#2723 Zbl 0221.57016

[Webster 1985] S. M. Webster, "Real submanifolds of $\mathbb{C}^{n}$ and their complexifications", pp. 6979 in Topics in several complex variables (Santa Cruz, Mexico, 1983), edited by E. Ramírez de Arellano and D. Sundararaman, Res. Notes in Math. 112, Pitman, Boston, 1985. MR 86k:32019 Zbl 0566.32014

[Whitney 1943] H. Whitney, "The general type of singularity of a set of $2 n-1$ smooth functions of $n$ variables", Duke Math. J. 10 (1943), 161-172. MR 4,193b Zbl 0061.37207

[Whitney 1944a] H. Whitney, "The self-intersections of a smooth $n$-manifold in $2 n$-space", Ann. of Math. (2) 45 (1944), 220-246. MR 5,273g Zbl 0063.08237

[Whitney 1944b] H. Whitney, "The singularities of a smooth $n$-manifold in (2n-1)-space", Ann. of Math. (2) 45 (1944), 247-293. MR 5,274a Zbl 0063.08238

[Whitney 1958] H. Whitney, "Singularities of mappings of Euclidean spaces", pp. 285-301 in Symposium internacional de topología algebraica (Mexico City, 1958), Universidad Nacional Autónoma de México and UNESCO, Mexico City, 1958. MR 20 \#4877 Zbl 0092.28401

Received December 5, 2004.

\section{ADAM COFFMAN}

DePartment of Mathematical SCIEnCES

Indiana Univ. - PURdue Univ. Fort WAYNe

2101 E. COLISEUM BLVD.

FORT WAYNE, IN 46805-1499

UNITED STATES

CoffmanA@ipfw.edu

http://www.ipfw.edu/math/Coffman/ 